\title{
Diffusion of finite-size particles in confined geometries
}

\author{
Maria Bruna • S. Jonathan Chapman
}

Received: 13 December 2012 / Accepted: 22 April 2013

\begin{abstract}
The diffusion of finite-size hard-core interacting particles in two- or three-dimensional confined domains is considered in the limit that the confinement dimensions become comparable to the particle's dimensions. The result is a nonlinear diffusion equation for the one-particle probability density function, with an overall collective diffusion that depends on both the excluded-volume and the narrow confinement. By including both these effects the equation is able to interpolate between severe confinement (for example, single-file diffusion) and unconfined diffusion. Numerical solutions of both the effective nonlinear diffusion equation and the stochastic particle system are presented and compared. As an application, the case of diffusion under a ratchet potential is considered, and the change in transport properties due to excluded-volume and confinement effects is examined.
\end{abstract}

Keywords Brownian motion · Fokker-Planck equation · Diffusion in confined geometries · Entropic effects · Stochastic simulations

\section{Introduction}

Transport of material under confined conditions occurs throughout nature and applications in industry. Examples include the transport of particles in biological cells, such as ion channels that conduct ions across the cell surface (Hille 2001) or intracellular cargo along microtubule filaments (Alberts et al 2002; Klumpp et al 2005), and in zeolites (Keil et al 2000). Similarly, confinement can be important in the diffusion of cells themselves (e.g. blood cells through microvessels, Pries et al 1996) and surface diffusion on the cell membrane, which is usually crowded with fixed and mobile obstacles (Nicolau Jr. et al 2007). Moreover, recent advances in nanotechnology have allowed the development of synthetic nanopores and microfluidic devices (Hänggi and Marchesoni 2009), which can be used for the sensing of single particles (such as small molecules, organic polymers, proteins, or enzymes)

Mathematical Institute, University of Oxford, 24-29 St. Giles', Oxford OX1 3LB, United Kingdom

E-mail: bruna@maths.ox.ac.uk·chapman@maths.ox.ac.uk 
and for studying chemical reactions, biomolecular recognition, and interactions at the nanoscale (Dekker 2007; Howorka and Siwv 2009). A common feature in these applications is the interplay between the particle motion (usually noisy) and the geometric constraints. An additional factor comes into play if the system contains a collective of interacting particles rather than an individual particle. The way in which these characteristics combine to produce the global behaviour is a crucial factor in the understanding of such systems. In many respects, these transport phenomena can be studied in terms of the canonical problem of geometrically constrained Brownian dynamics (Burada et al 2009).

When considering a theoretical model of particle diffusion in confined environments, there are three important modelling decisions to make. First, one must decide on the most appropriate representation of the particle diffusion and interactions (with other particles and the confining walls). For example, a common approach is to use a lattice-based random walk model with exclusion (Plank and Simpson 2012), that is, to assume that the motion of particles is restricted to taking place on a lattice and that any attempted move to an occupied site is aborted. An alternative approach is to consider a lattice-free random walk, in which the individual particle movements are not restricted to a lattice. It this case, excluded-volume interactions can be taken into account by assuming particles are hard spheres which cannot overlap each other, thus considering a Brownian motion of hard spheres (Bruna and Chapman 2012b). While in some cases a lattice-based model is more suitable for the particular application, in general the lattice-free approach is more realistic (Plank and Simpson 2012) and the choice of an on-lattice model is for technical convenience only.

The second modelling decision concerns the level of description, that is, whether to use an individual-based model or a population-based model. In the first case, the system of diffusing and interacting particles is represented with a stochastic model that describe the dynamics and interactions of each particle explicitly. This is typically a computationally intensive approach, involving many statistically identical realisations of the stochastic simulation to develop insight into the population-level dynamics. In contrast, the population-based model consists of a continuum description of the system in the form of a partial differential equation (PDE) for the population density of individuals. The continuum model tends to be easier to solve and analyse and can be particularly useful when, for large systems of interacting particles, discrete models become computationally intractable. However, the challenge is to predict the correct PDE description of a given system of interacting particles, and, as a result, many population-based models are described phenomenologically at the continuum level rather than derived from the underlying particle transport process. For example, while it is well-understood that a non-interacting Brownian motion is associated a linear diffusion PDE at the population-level, it is not so straightforward to predict how excluded-volume interactions at the discrete level emerge in the PDE model. As pointed out in Plank and Simpson (2012), the ability to represent mathematically both the individual-level details and the population-level description of a stochastic particle system is important because many experimental observations involve data at both levels for the same system. As a result, if we are to use both the individual-based and the population-based models of the same system, the link between the two must be fully understood to ensure that both models are consistent with each other. 
Finally, the third consideration has to do with the way confinement is included in the model. It is important to note that the idea of confinement is inevitably relative the particle's characteristic size. A common approximation of confinement situations which is applicable when the particles are much smaller than the channel width is to ignore steric interactions between particles (assuming they are simply points) and only consider the geometric effects of the confining environment (Burada et al 2009). For example, the diffusion of point particles in a (narrow) tube of varying cross-section can be approximated by an effective one-dimensional diffusion equation known as the Fick-Jacobs equation (Jacobs 1967; Reguera and Rubí 2001). Another example in which particle interactions are omitted can be found in the Brownian ratchet models of molecular motors (Muñoz-Gutiérrez et al 2012; Eichhorn et al 2002), which take the form of a one-dimensional diffusion under a periodic potential and tilting force.

The opposite limit is single-file diffusion (Henle et al 2008), in which the finitesize of particles is taken into account but the confinement is so extreme that particles cannot diffuse past each other (imagine a channel of width equal to the diameter of particles). Mathematically this problem is modelled as a one-dimensional domain with hard-core interacting particles (hard rods) and has been widely studied (see, for example, Lizana and Ambjörnsson 2009; Bodnar and Velázquez 2005).

Both of these limits are extremes. The distinguished limit in which the finitesize interactions are important but the confinement is not so extreme that particles cannot pass one another has received little attention; one notable exception is the exclusion process on a lattice in Henle et al (2008).

\subsection{Aim of this paper}

This work introduces a theoretical framework for studying particle diffusion processes in confined environments. Rather than attempting to answer a particular question related to one of the applications presented earlier, here we are interested in developing a technique to tackle the common first steps in any of these such problems. Following the three considerations outlined above, we are interested in a lattice-free approach, in deriving the population-level model systematically from the individual-based model, and in an intermediate level of confinement. To this end, we consider the evolution of a system of $N$ identical hard spheres in a confined domain, in the limit that the confinement dimensions become comparable to the particle dimensions. In this setting, the finite size of particles is important not only for particle-particle interactions, but also for interactions with the domain walls. We consider in particular three confinement scenarios: a two-dimensional channel, a three-dimensional square channel, and two close parallel plates. However, since our approach is systematic, our model can be extended to other geometries.

The key idea is that the system will reach equilibrium in the confined directions quickly, leading to an effective diffusion of reduced dimension in the unconfined directions only. With this in mind, the solution procedure consists of two steps: first, to reduce the model of $N$ interacting particles to a model for the evolution of the one-particle marginal density, as we did in Bruna and Chapman (2012b); and second, to reduce the resulting model from a $d$-dimensional confined domain to an effective one-dimensional axial model in the case of a narrow channel, or to an effective two-dimensional planar model in the case of parallel plates. 
1.2 Plan of this paper

The work is organised as follows: in the next section we will introduce the problem setup, illustrate how the problem simplifies in the case of point particles and present the main result of this work, a population-level PDE model for the diffusion of hard spheres as a function of a confinement parameter, given by equation (10). In the third section we examine how our model interpolates between the different limiting cases of confinement. In Section 4 we explore numerical solutions of our PDE model and compare them with stochastic simulations of the particle-based model and numerical solutions of the limiting models. Finally, the fifth section will be devoted to the derivation of (10) for a two-dimensional channel.

\section{The model}

2.1 The setup: drift-diffusion in confined geometries

We consider a population of $N$ identical particles diffusing in a bounded domain $\Omega \subset \mathbb{R}^{d}(d=2,3)$, interacting with each other and the domain walls with a repulsive hard-core potential, and in the presence of an external force. We work in the dimensionless problem by scaling space with a typical unconfined dimension $L$, time with $L^{2} / D_{0}$ where $D_{0}$ is the constant molecular diffusion coefficient, and force with $\gamma D_{0} / L$ where $\gamma$ is the frictional drag coefficient. We assume particles are spherical with nondimensional diameter $\epsilon \ll 1$.

Assuming the overdamped limit, the stochastic dynamics of the system is described by a set of stochastic Langevin equations

$$
\mathbf{X}_{i}(t)=\mathbf{f}\left(\mathbf{X}_{i}(t)\right) \underline{t}+\sqrt{2} \mathbf{W}_{i}(t), \quad i=1, \ldots N,
$$

where $\mathbf{X}_{i}(t) \in \Omega$ denotes the centre of particle $i$ at time $t \geq 0$, $\mathbf{f}$ is the dimensionless external force (or drift) and $\mathbf{W}_{i}$ are $N$ independent $d$-dimensional standard Brownian motions. We note that by writing $\mathbf{f}\left(\mathbf{X}_{i}(t)\right)$ we are assuming that the force acting on the $i$ th particle only depends on its own position, thus excluding forces such as the electromagnetic force which would depend on the positions of all the particles $\vec{X}=\left(\mathbf{X}_{1}, \ldots, \mathbf{X}_{N}\right)$. We suppose that the initial positions $\mathbf{X}_{i}(0)$ are random and identically distributed. Note that, because of the finite size of particles, we have the set of constraints $\left\|\mathbf{X}_{i}-\mathbf{X}_{j}\right\| \geq \epsilon$ for $i \neq j$, so that the system of SDEs (11) is coupled.

The Langevin system (1) is equivalent to the Fokker-Planck equation for the joint probability density $P(\vec{x}, t)$ of the $N$ particles to be found at the position $\vec{x}=\left(\mathbf{x}_{1}, \ldots, \mathbf{x}_{N}\right) \in \Omega^{N}$ at time $t$, given by

$$
\frac{\partial P}{\partial t}(\vec{x}, t)=\nabla_{\vec{x}} \cdot\left[\nabla_{\vec{x}} P-\vec{F}(\vec{x}) P\right]
$$

where $\vec{\nabla}_{\vec{x}}$ and $\vec{\nabla}_{\vec{x}}$. respectively stand for the gradient and divergence operators with respect to the $N$-particle position vector $\vec{x}$ and $\vec{F}(\vec{X})=\left(\mathbf{f}\left(\mathbf{x}_{1}\right), \ldots, \mathbf{f}\left(\mathbf{x}_{N}\right)\right)$ is the total drift vector. Because of excluded-volume effects, the domain of definition of (2) (or configuration space) is not $\Omega^{N}$ but its hollow form $\Omega_{\epsilon}^{N}=\Omega^{N} \backslash \mathcal{B}_{\epsilon}$, where $\mathcal{B}_{\epsilon}=\left\{\vec{x} \in \Omega^{N}: \exists i \neq j\right.$ such that $\left.\left\|\mathbf{x}_{i}-\mathbf{x}_{j}\right\| \leq \epsilon\right\}$ is the set of all illegal 
configurations (with at least one overlap). On the contact surfaces $\partial \Omega_{\epsilon}^{N}$ we have the reflecting boundary condition

$$
0=\left[\nabla_{\vec{x}} P-\vec{F}(\vec{x}) P\right] \cdot \vec{n},
$$

where $\vec{n} \in \mathcal{S}^{d N-1}$ denotes the unit outward normal. Finally, since the particles are initially identically distributed, the initial probability density $P(\vec{x}, 0)=P_{0}(\vec{x})$ is invariant to permutations of the particle labels. The form of (2) then means that $P$ itself is invariant to permutations of the particle labels for all times.

We suppose that $\Omega$ is a confined domain, with $k<d$ confinement dimensions which are comparable to $\epsilon$. We introduce $d_{e}=d-k$ as the effective dimensionality of the problem. In particular, we shall consider the following cases:

$\diamond \quad($ NC2 $)$ Two-dimensional narrow channel $\left(d=2, k=1\right.$ and $\left.d_{e}=1\right)$ :

$$
\Omega=\left[-\frac{1}{2}, \frac{1}{2}\right] \times\left[-\frac{H}{2}, \frac{H}{2}\right] .
$$

$\diamond \quad$ (NC3) Three-dimensional narrow channel $\left(d=3, k=2\right.$ and $\left.d_{e}=1\right)$ :

$$
\Omega=\left[-\frac{1}{2}, \frac{1}{2}\right] \times\left[-\frac{H}{2}, \frac{H}{2}\right] \times\left[-\frac{H}{2}, \frac{H}{2}\right] .
$$

$\diamond \quad(\mathbf{P P})$ Two parallel plates $\left(d=3, k=1\right.$ and $\left.d_{e}=2\right)$ :

$$
\Omega=\left[-\frac{1}{2}, \frac{1}{2}\right] \times\left[-\frac{1}{2}, \frac{1}{2}\right] \times\left[-\frac{H}{2}, \frac{H}{2}\right],
$$

where $H=\mathcal{O}(\epsilon)$ is the confinement parameter. We note that $H \geq 0$, with $H=0$ allowed since $\Omega$ is the volume available to the particles' centres. In the case of a narrow-channel, when $H<\epsilon$ particles cannot pass each other. We assume that the volume fraction is small; since $|\Omega|=\mathcal{O}\left(\epsilon^{k}\right)$ this implies that $N \epsilon^{d_{e}} \ll 1$.

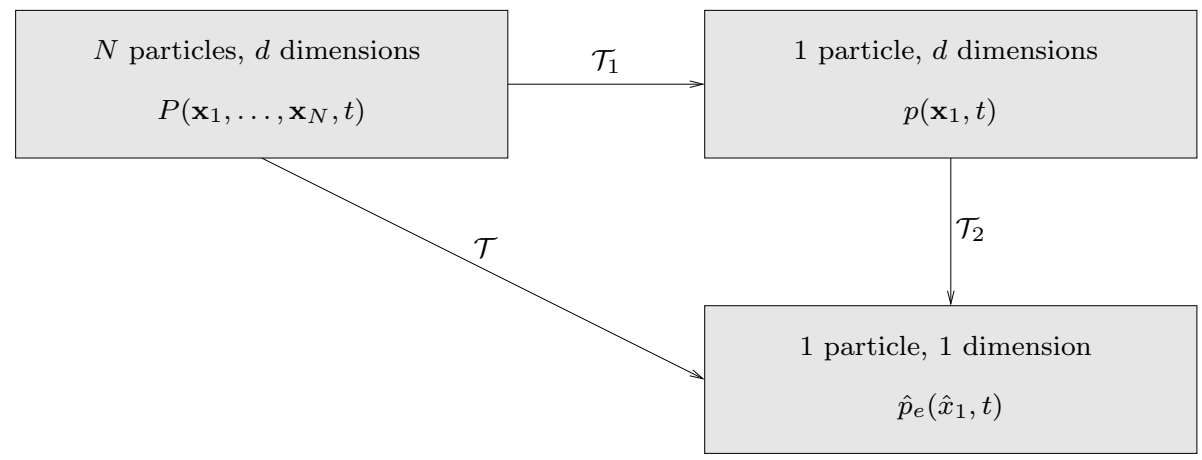

Figure 1 Schematic of the problem solution steps for $d_{e}=1$ [narrow-channel cases (NC2) and (NC3)]. The goal is transformation $\mathcal{T}$, to obtain an effective one-dimensional equation along the channel for the marginal density of one particle. We achieve this with the combined steps $\mathcal{T}_{1}$ followed by $\mathcal{T}_{2}$.

The high-dimensional diffusion problem (2) will be reduced to an effective $d_{e}$-dimensional transport model in two steps (see Figure 1). First, as we did in Bruna and Chapman (2012b), the dimensions can be reduced from $d N$ to $d$ (individual to population-level description) by looking at the marginal density function 
of one particle (the first particle, say) given by $p\left(\mathbf{x}_{1}, t\right)=\int P(\vec{x}, t) \mathrm{d} \mathbf{x}_{2} \cdots \mathrm{d} \mathbf{x}_{N}$ (the particle choice is unimportant since all the particles are identical). Second, we will exploit the geometry of the domain $\Omega$ to further reduce the dimensionality by $k$, the number of confining dimensions. To this end, we will introduce the narrow-domain variables and obtain, from the $d$-dimensional density $p(\mathbf{x}, t)$ a reduced effective density $\hat{p}_{e}\left(\boldsymbol{x}_{e}, t\right)$, with $\boldsymbol{x}_{e} \in \mathbb{R}^{d_{e}}$. For cases (NC2) and (NC3), the effective density $\hat{p}_{e}$ will be a one-dimensional density $\hat{p}_{e}(x, t)$ along the channel axis. For (PP), it will be an effective two-dimensional density on the plane, $\hat{p}_{e} \equiv \hat{p}_{e}(x, y, t)$.

For the sake of clarity we illustrate the derivation for the two-dimensional case (NC2) for both point and finite-size particles; the extension to the threedimensional cases follows similarly and the respective models are only given in a summarised form.

\subsection{Point particles}

We begin by considering the case of point particles, for which the first reduction $\mathcal{T}_{1}$ in Figure 1 from $N$ to one particle is straightforward. Since the particles are independent, $P(\vec{x}, t)=\prod_{i=1}^{N} p\left(\mathbf{x}_{i}, t\right)$, and

$$
\begin{aligned}
& \frac{\partial p}{\partial t}(\mathbf{x}, t)=\boldsymbol{\nabla}_{\mathbf{x}} \cdot\left[\boldsymbol{\nabla}_{\mathbf{x}} p-\mathbf{f}(\mathbf{x}) p\right] \quad \text { in } \quad \Omega, \\
& 0=\left[\boldsymbol{\nabla}_{\mathbf{x}} p-\mathbf{f}(\mathbf{x}) p\right] \cdot \hat{\mathbf{n}} \quad \text { on } \quad \partial \Omega,
\end{aligned}
$$

where $\hat{\mathbf{n}}$ is the outward unit normal to $\partial \Omega$. Thus we move to the second model reduction $\mathcal{T}_{2}$ which is applied to (4). Using the definition of $\Omega$ (3a), we want to exploit the smallness of $H$. We introduce a change of variables to the narrowdomain variables, which consist of rescaling by $\epsilon$ the variables corresponding to the confined dimension:

$$
x=\hat{x}, \quad y=\epsilon \hat{y} .
$$

Introducing $h$ such that $H=\epsilon h$, the domain $\Omega$ transforms into $\omega=\left[-\frac{1}{2}, \frac{1}{2}\right] \times$ $\left[-\frac{h}{2}, \frac{h}{2}\right]$. In the rescaled domain, we define $\hat{p}(\hat{\mathbf{x}}, t)=\epsilon p(\mathbf{x}, t)$. (The factor of $\epsilon$ is introduced so that both $p$ and $\hat{p}$ integrate to one in their respective domains $\Omega$ and $\omega$. .) Then (4) becomes

$$
\epsilon^{2} \frac{\partial \hat{p}}{\partial t}(\hat{\mathbf{x}}, t)=\epsilon^{2} \frac{\partial}{\partial \hat{x}}\left(\frac{\partial \hat{p}}{\partial \hat{x}}-f_{1}(\hat{x}, \epsilon \hat{y}) \hat{p}\right)+\frac{\partial}{\partial \hat{y}}\left(\frac{\partial \hat{p}}{\partial \hat{y}}-\epsilon f_{2}(\hat{x}, \epsilon \hat{y}) \hat{p}\right)
$$

in $\omega$, with boundary conditions

$$
\begin{array}{llrl}
\frac{\partial \hat{p}}{\partial \hat{x}}=f_{1}(\hat{x}, \epsilon \hat{y}) \hat{p} & \text { on } & \hat{x} & = \pm \frac{1}{2} \\
\frac{\partial \hat{p}}{\partial \hat{y}}=\epsilon f_{2}(\hat{x}, \epsilon \hat{y}) \hat{p} & \text { on } & \hat{y}= \pm \frac{h}{2}
\end{array}
$$

where $f_{1}$ and $f_{2}$ are respectively the horizontal and vertical components of the external force $\mathbf{f}$. Expanding $\hat{p}$ in powers of $\epsilon$, Taylor-expanding $f_{1}$ and $f_{2}$ around $(\hat{x}, 0)$, and solving (6a) with the boundary condition (6c) gives, at leading order, 
that $\hat{p}$ is independent of $\hat{y}$. Integrating (6a) over the channel's cross section and using (6c) we find that, to $\mathcal{O}(\epsilon)$

$$
\frac{\partial \hat{p}_{e}}{\partial t}(\hat{x}, t)=\frac{\partial}{\partial \hat{x}}\left(\frac{\partial \hat{p}_{e}}{\partial \hat{x}}-f_{1}(\hat{x}, 0) \hat{p}_{e}\right) \quad \hat{x} \in[-1 / 2,1 / 2]
$$

where $\hat{p}_{e}=\int_{-h / 2}^{h / 2} \hat{p} \mathrm{~d} \hat{y}$ is the effective one-dimensional density along the channel. This equation is complemented with no-flux boundary conditions at $\hat{x}= \pm 1 / 2$. Equation (7) can be generalised to three-dimensional geometries as

$$
\frac{\partial \hat{p}_{e}}{\partial t}\left(\hat{\mathbf{x}}_{e}, t\right)=\nabla_{\hat{\mathbf{x}}_{e}} \cdot\left[\nabla_{\hat{\mathbf{x}}_{e}} \hat{p}_{e}-\mathbf{f}_{e}\left(\hat{\mathbf{x}}_{e}\right) \hat{p}_{e}\right] \quad \hat{\mathbf{x}}_{e} \in \omega_{e}
$$

with no-flux boundary conditions on $\partial \omega_{e}$, where $\hat{\mathbf{x}}_{e} \in \omega_{e}$ are the coordinates in the effective domain (i.e. the one-dimensional axis for (NC3) as in (7), or the twodimensional plane for $(\mathrm{PP})$ ). The effective drift $\mathbf{f}_{e}$ is the projection of the full drift vector onto the effective domain $\omega_{e}$. The initial condition is $\hat{p}_{e}\left(\hat{\mathbf{x}}_{e}, 0\right)=\hat{p}_{0}\left(\hat{\mathbf{x}}_{e}\right)$, where $\hat{p}_{0}\left(\hat{\mathbf{x}}_{e}\right)=\int_{\Omega^{N}} P_{0}(\vec{x}) \delta\left(\hat{\mathbf{x}}_{e}-\mathbf{x}_{1, e}\right) \mathrm{d} \vec{x}$.

A common extension to (7) is to suppose that the channel has a non-constant cross section, $h=h(x)$. The simplest model is the Fick-Jacobs equation (Jacobs 1967), which in our notation reads

$$
\frac{\partial \hat{p}_{e}}{\partial t}(\hat{x}, t)=\frac{\partial}{\partial \hat{x}}\left[h(\hat{x}) \frac{\partial}{\partial \hat{x}}\left(\frac{\hat{p}_{e}}{h(\hat{x})}\right)-f_{1}(\hat{x}, 0) \hat{p}_{e}\right],
$$

and is valid for $\epsilon h^{\prime}(x)$ small. Generalisations to this equation to account for the channel curvature (a higher-order term) have been given in (Reguera and Rubil 2001). The key step in deriving (9) is to assume that the full two- or threedimensional probability density $\hat{p}(\hat{\mathbf{x}}, t)$ is at equilibrium in the transverse direction, that is, it is assumed to factorise as $\hat{p}(\hat{\mathbf{x}}, t) \approx \hat{p}_{e}(\hat{x}, t) \rho(\hat{\mathbf{x}})$, where $\rho(\hat{\mathbf{x}})$ is the local equilibrium distribution of $\hat{y}$ (and $\hat{z}$, for $d=3$ ), conditional on a given $\hat{x}$ (the normalised Boltzmann-Gibbs probability density); see Zwanzig (1992).

In what follows, we keep $h$ constant since the inclusion of a variable channel width in the analysis for finite-size particles is not straightforward.

\subsection{Finite-size particles}

We now describe the main result of this paper: the model of the effective dynamics in a confined domain for the drift-diffusion of finite-size particles. Using a similar technique to our previous work Bruna and Chapman (2012b), we are able to reduce the Fokker-Planck equation (2) for the joint probability density $P(\vec{x}, t)$ of $N$ interacting finite-size particles in a confined domain $\Omega$ to the following effective equation for the marginal density $\hat{p}_{e}\left(\hat{\boldsymbol{x}}_{e}, t\right)$ :

$$
\frac{\partial \hat{p}_{e}}{\partial t}\left(\hat{\boldsymbol{x}}_{e}, t\right)=\boldsymbol{\nabla}_{\hat{\boldsymbol{x}}_{e}} \cdot\left\{\left[1+(N-1) \epsilon^{d_{e}} \alpha_{h} \hat{p}_{e}\right] \boldsymbol{\nabla}_{\hat{\boldsymbol{x}}_{e}} \hat{p}_{e}-\boldsymbol{f}_{e}\left(\hat{\boldsymbol{x}}_{e}\right) \hat{p}_{e}\right\},
$$

for $\hat{\boldsymbol{x}}_{e} \in \omega_{e} \subset \mathbb{R}^{d_{e}}$, where $d_{e}$ are the effective dimensions of the reduced domain $\omega_{e}$. The coefficient $\alpha_{h}$, which depends on the geometry of the problem, determines how the excluded volume varies with the confinement parameter $h$. This equation is complemented with no-flux boundary conditions on $\partial \omega_{e}$ and initial data $\hat{p}_{e}\left(\hat{\boldsymbol{x}}_{e}, 0\right)=$ $\hat{p}_{0}\left(\hat{\boldsymbol{x}}_{e}\right)$. Below we specify the coefficient $\alpha_{h}$ for some specific cases. 
1. Two-dimensional channel of width $h(\mathrm{NC} 2), d_{e}=1$ :

$$
\alpha_{h}=\frac{1}{h^{2}}\left[\pi h-\frac{4}{3}+\Theta(1-h)\left(\frac{2}{3}\left(2+h^{2}\right) \sqrt{1-h^{2}}-2 h \arccos (h)\right)\right]
$$

where $\Theta(x)$ is the Heaviside step function,

$$
\Theta(x)= \begin{cases}0 & x<0 \\ 1 & x \geq 0\end{cases}
$$

2. Three-dimensional channel of cross-section $h \times h(\mathrm{NC} 3), d_{e}=1$ :

$$
\alpha_{h}=\frac{1}{h^{4}}\left[\Theta(h-1)\left(\frac{4 \pi}{3} h^{2}-\pi h+\frac{8}{15}\right)+\Theta(1-h) m(h)\right],
$$

where

$$
m(h)=s(h)+\left\{\begin{array}{lr}
\sigma_{a}(h) & 0 \leq h \leq \frac{\sqrt{5}-1}{2}, \\
\sigma_{b}(h) & \frac{\sqrt{5}-1}{2}<h \leq \frac{1}{\sqrt{2}} \\
0 & \frac{1}{\sqrt{2}}<h
\end{array}\right.
$$

with

$$
\begin{aligned}
s(h)=\frac{8}{15} & +\frac{2}{15} \sqrt{1-h^{2}}\left(2 h^{4}-9 h^{2}-8\right)-\frac{\pi}{3} h\left(h^{4}-6 h^{2}+4 h-3\right)-2 h \arcsin (h), \\
\sigma_{a}(h)= & \frac{2}{15} \sqrt{1-2 h^{2}}\left(h^{4}+9 h^{2}+4\right)+\frac{\pi}{12} h\left(3 h^{4}-18 h^{2}+16 h-9\right) \\
& +\frac{1}{6} h^{3}\left(h^{2}-6\right) \operatorname{arccot}\left(\frac{2 h \sqrt{1-2 h^{2}}}{1-3 h^{2}}\right)-\frac{4}{3} h^{2} \operatorname{arccot}\left(\frac{1-2 h^{2}-h^{4}}{2 h^{2} \sqrt{1-2 h^{2}}}\right) \\
& -\frac{1}{2} h \operatorname{arccot}\left(\frac{2 h\left(1-2 h^{2}\right)^{3 / 2}+2 h \sqrt{1-h^{2}}\left(3 h^{2}-1\right)}{1-5 h^{2}+6 h^{4}+4 h^{2} \sqrt{\left(1-2 h^{2}\right)\left(1-h^{2}\right)}}\right) \\
& +h \arcsin (h)-\frac{1}{3} h\left(h^{4}-6 h^{2}-3\right) \arcsin \left(\frac{h}{\sqrt{1-h^{2}}}\right), \\
\sigma_{b}(h)= & \frac{2}{15} \sqrt{1-2 h^{2}}\left(h^{4}+9 h^{2}+4\right)+\frac{\pi}{12} h\left(2 h^{4}-12 h^{2}+8 h-3\right) \\
& +\frac{1}{3} h^{3}\left(h^{2}-6\right) \operatorname{arccot}\left(\frac{2 h \sqrt{1-2 h^{2}}}{1-3 h^{2}}\right)+\frac{4}{3} h^{2} \arctan \left(\frac{1-2 h^{2}-h^{4}}{2 h^{2} \sqrt{1-2 h^{2}}}\right) \\
& +\frac{1}{2} h \operatorname{arccot}\left(\frac{4 h \sqrt{1-2 h^{2}}\left(3 h^{2}-1\right)}{1-10 h^{2}+17 h^{4}}\right) .
\end{aligned}
$$

3. Three-dimensional parallel plates a distance $h$ apart $(\mathrm{PP}), d_{e}=2$ :

$$
\alpha_{h}=\frac{\pi}{6 h^{2}}\left[\left(h^{2}\left(6-h^{2}\right)\right) \Theta(1-h)+(8 h-3) \Theta(h-1)\right] .
$$




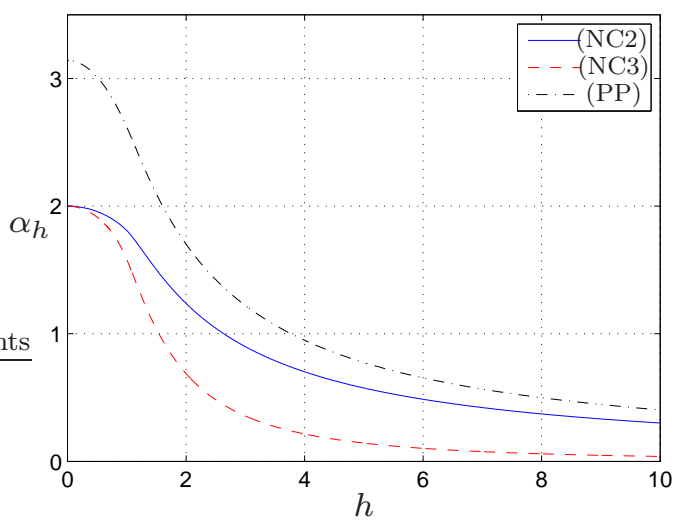

Figure 2 Excluded-volume coefficient $\alpha_{h}$ as a function of the confinement parameter $h$ in three cases: two-dimensional channel (NC2) (11), three-dimensional channel (NC3) 12), and parallel plates (PP) (14).

The coefficient $\alpha_{h}$ corresponding to cases (NC2), (NC3), and (PP) is plotted in Figure 2

Equation (10) describes the probability density for finding the first particle at position $\hat{\mathbf{x}}_{e}$ at time $t$. Since the original system (2) is invariant to permutations of the particle labels, the marginal density function of any other particle is the same. Thus the probability distribution function for finding any particle at position $\hat{\boldsymbol{x}}_{e}$ at time $t$ is simply $N \hat{p}_{e}$.

In Figure 3 we sketch the narrow-channel domain (rescaled by $\epsilon$ ) for various heights $h$. The physical domain (of width $h+1$ in the narrow-domain variables) is delimited by the solid black lines, while the configuration domain (of width $h$ ) corresponds to the yellow shaded region delimited by dot-dash lines.

The nonlinear diffusion term in (10) is proportional to the effective excluded volume created by the remaining $(N-1)$ particles as well as the domain walls after the dimensional reduction. For example, in the (NC2) case $\epsilon \alpha_{h}$ is the effective onedimensional excluded interval, corresponding to the excluded area divided by the height of the cross section available to a particle centre (see Figure 3 ). When $h=0$, a particle of diameter $\epsilon$ excludes an interval of $2 \epsilon$ (this explains why $\alpha_{h}=2$ for $h=0$; see Figure 2). As the channel width increases, the value $\epsilon \alpha_{h}$ decreases since the whole width of the channel is not always excluded by a given particle. As $h$ gets large $\epsilon \alpha_{h}$ gives the ratio of the area excluded by the particle, $\pi \epsilon^{2}$, to the cross section height $\epsilon h$, so that $\alpha_{h} \sim \pi / h$ as $h \rightarrow \infty$ [see (11)].

While $\alpha_{h}$ gives the effective excluded volume after dimensional reduction, the actual excluded volume is proportional to $h \alpha_{h}$. This is plotted in Figure 4 [along with the corresponding expressions for (NC3) and (PP)].

It is clear from Figure 3 that the excluded volume due to a particle varies depending on its position in the channel's cross-section: in (NC2), while a particle excludes an area of $\pi$ when it is far from the channel walls, it only excludes half of this area when in contact with the channel walls (less if $h<1$ ). This effect, known as an entropic effect, implies that the average excluded area over possible locations across the channel width decreases as the channel narrows $(h \rightarrow 0)$. As 

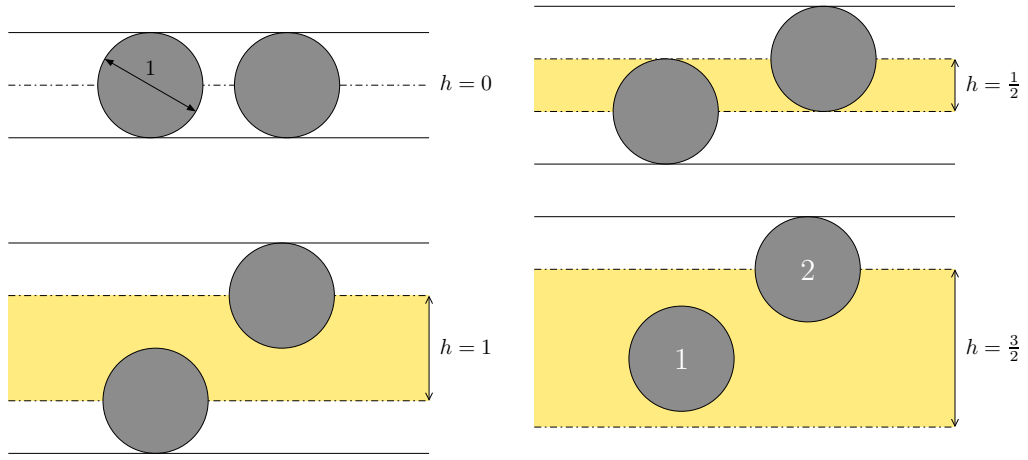

Figure 3 Sketch of the channel domain (shaded in yellow) for different values of $h$ with particles of diameter one (note that here we are depicting the actual particles, not their excludedarea which has radius one). Single-file channel for $0 \leq h<1$ (with $h=0$ being the extreme case in which particles can only move in the axial direction). When $h=1$ particles can just pass each other, and for $h>1$ (bottom row) particles can more easily change order.

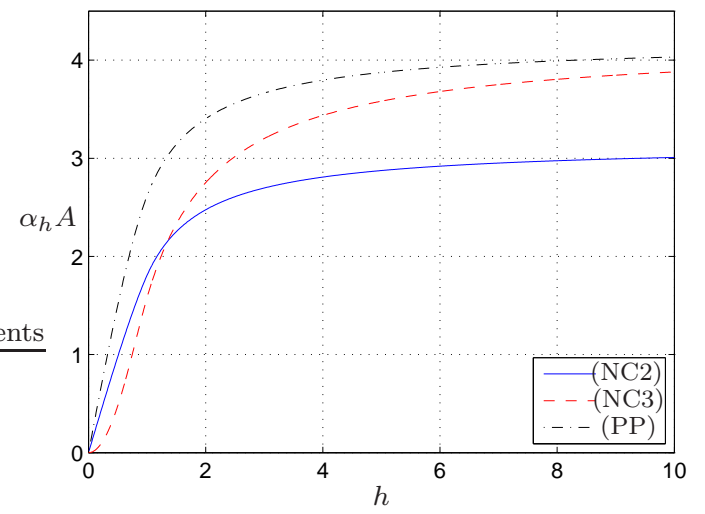

Figure 4 Excluded volume $\alpha_{h} A$ as a function of the confinement parameter $h$ in three cases: two-dimensional channel ( $\mathrm{NC} 2)(A=h)$, three-dimensional channel (NC3) $\left(A=h^{2}\right)$, and parallel plates $(\mathrm{PP})(A=h)$.

the channel width $h$ grows, the boundary effects in which the excluded area is reduced contribute less and less to the average value, implying that the average excluded area tends to the constant value $\pi$ as $h \rightarrow \infty$, which corresponds to the "bulk" excluded area. This is confirmed in Figure 4. Similarly, $\alpha_{h}$ tends to $4 \pi / 3$ for the three dimensional cases as this is the rescaled excluded volume (the volume of the unit sphere). As $h \rightarrow 0$ the average excluded area $h \alpha_{h} \rightarrow 0$ since in the extreme confinement cases almost all of the actual excluded area lies outside the domain available to a particle's centre. 


\subsubsection{Effective equation for the volume concentration}

In our derivation of (10) we do not require $N$ to be large: in fact, equation (10) is valid for any $N$ (as long as the volume fraction is small), so that one could set $N=1$ or 2 if required. This equation gives the probability of finding a particle at a given position at a given time. However, for large $N$ such that $N-1 \approx N$ we can introduce the volume concentration $\hat{c}_{e}=\phi \hat{p}_{e}$, where $\phi$ is the total volume fraction of particles, and rewrite equation (10) as an equation for the concentration of particles in the system:1

$$
\frac{\partial \hat{c}_{e}}{\partial t}\left(\hat{\boldsymbol{x}}_{e}, t\right)=\nabla_{\hat{\boldsymbol{x}}_{e}} \cdot\left[\left(1+g_{h} \hat{c}_{e}\right) \boldsymbol{\nabla}_{\hat{\boldsymbol{x}}_{e}} \hat{c}_{e}-\boldsymbol{f}_{e}\left(\hat{\boldsymbol{x}}_{e}\right) \hat{c}_{e}\right]
$$

with

$$
\begin{aligned}
(\mathrm{NC} 2): & g_{h}=\frac{4}{\pi}(h+1) \alpha_{h}, & \phi & =\frac{N \pi \epsilon}{4(h+1)}, \\
(\mathrm{NC} 3): & g_{h}=\frac{6}{\pi}(h+1)^{2} \alpha_{h}, & \phi & =\frac{N \pi \epsilon}{6(h+1)^{2}}, \\
(\mathrm{PP}): & g_{h}=\frac{6}{\pi}(h+1) \alpha_{h}, & \phi & =\frac{N \pi \epsilon^{2}}{6(h+1)} .
\end{aligned}
$$

These expressions for $g_{h}$ are plotted in Figure 5. We note all three have a finite value as both $h \rightarrow 0$ and $h \rightarrow \infty$ and, most importantly, that they have a relative maximum at $h=h^{*}$, where $h^{*}$ is slightly greater than one. In particular, $h^{*}=1.47$, $h^{*}=1.28$, and $h^{*}=1.2$ for $(\mathrm{NC} 2)$, (NC3), and (PP) respectively.

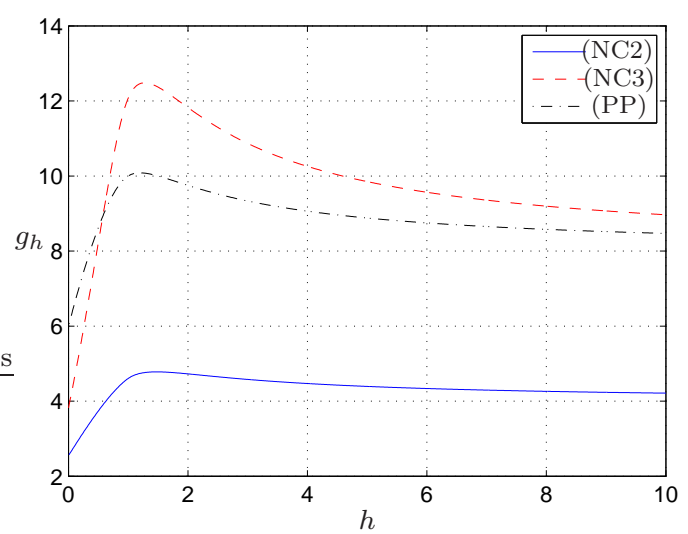

Figure 5 Coefficient $g_{h}$ as a function of the confinement parameter $h$ in three cases: twodimensional channel (NC2), three-dimensional channel (NC3), and parallel plates (PP).

The presence of this relative maximum at a fixed volume fraction is interesting, as it implies an optimal ratio between the particles' size and the confinement

\footnotetext{
1 Note the factors $(1+h)$ in $\phi$ : this is because $\phi$ is the total volume of particles divided by the actual volume of the channel, not the volume available to a particle's centre.
} 
dimension at which excluded-volume effects, and thus the effective transport, are maximised. In terms of the physical domains, it corresponds to a narrow domain around 2.2 to 2.5 times wider than the particles' diameter, so that two particles can just diffuse side by side in the channel.
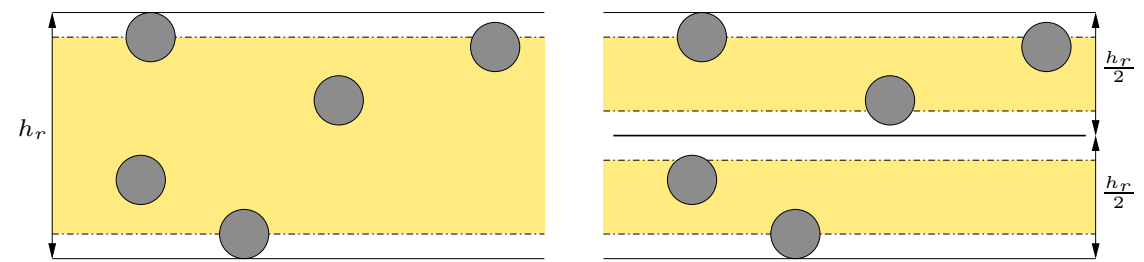

Figure 6 Sketch of channel of width $5 \epsilon$ with and without an intermediate wall that creates two lanes. The domain available to the particles centres' in each case is shaded in yellow. Left: one narrow channel with $h=4$. Right: two narrow channels each with $h=1.5$ (roughly equal to $h^{*}$ for maximal exclusion effects).

Thus the theory predicts that diffusive transport in a (NC2) channel of width $5 \epsilon(h=4)$ may be increased by dividing the channel into two sub-channels of width $2.5 \epsilon(h=1.5)$, as shown in Figure 6. This operation gives an increase in $g_{h}$ of $7 \%$. The increase is more dramatic in the three-dimensional case: if a (NC3) square channel has an original width and depth of $4.6 \epsilon$ (so that $h=3.6$ ), then subdividing it into four identical channels of width $2.3 \epsilon$ (so that each has $h=1.3$ ) gives a relative increase in $g_{h}$ is of $19 \%$.

\section{Limiting cases: from single-file diffusion to unconfined diffusion}

We have briefly discussed the limiting behaviour of $\alpha_{h}$ as $h \rightarrow 0$ and $h \rightarrow \infty$ above. Here we examine these limits in equation (10), and check that they agree with existing results. For a channel of width $h$ (NC2 or NC3) equation (10) interpolates between two limiting cases: a single-file channel $(h \rightarrow 0)$ and an unconfined two$(\mathrm{NC} 2)$ or three-dimensional (NC3) domain $(h \rightarrow \infty)$. For the (PP) case, the limit $h \rightarrow 0$ gives an (unconfined) two-dimensional diffusion, while the limit $h \rightarrow \infty$ gives three-dimensional unconfined diffusion. Finally, the extension of the square cross section (NC3) to a rectangular cross section $h \times m$ can be used to interpolate between (NC2) (as $m \rightarrow 0)$ and (PP) (as $m \rightarrow \infty)$.

In this section we will examine these limits by comparing the limiting behaviour of our model (10) with the limiting problem of diffusion of hard spheres in $\mathbb{R}^{d}$ for $d=1,2,3$. This problem has been studied extensively, especially in the onedimensional case, which is known as single-file diffusion Lizana and Ambiörnsson 2009). For the cases $d=2,3$ we will use the results from our previous work of unconfined diffusion of hard spheres (Bruna and Chapman 2012b). 
3.1 Limit to an unconfined domain: $h \rightarrow \infty$

As $h$ increases we expect the boundary effects contained in $\alpha_{h}$ to vanish and to recover the "bulk" or unconfined equation found in Bruna and Chapman (2012b):

$$
\frac{\partial p}{\partial t}(\mathbf{x}, t)=\nabla_{\mathbf{x}} \cdot\left\{\left[1+(N-1) \alpha \epsilon^{d} p\right] \nabla_{\mathbf{x}} p-\mathbf{f}(\mathbf{x}) p\right\} \quad \text { in } \quad \Omega,
$$

where $\Omega \subset \mathbb{R}^{d}$ as given in (3), $\alpha=\pi$ for $d=2$ and $\alpha=4 \pi / 3$ for $d=3$ (or $\alpha=2(d-1) \pi / d$ for $d=2,3)$. It is important to note that this equation is only valid for $H=\mathcal{O}(1)$ (that is, when $\Omega$ has volume order one).

In order to take the limit $h \rightarrow \infty$ in our model (10), it is convenient to use the original density $\hat{p}$ in $\mathbb{R}^{d}$ rather than the effective density $\hat{p}_{e}$. In other words, we consider the following equation for $\hat{p}=\hat{p}_{e} / A$ (where $A$ is the cross-sectional area):

$$
\frac{\partial \hat{p}}{\partial t}\left(\hat{\boldsymbol{x}}_{e}, t\right)=\boldsymbol{\nabla}_{\hat{\boldsymbol{x}}_{e}} \cdot\left\{\left[1+(N-1) \alpha_{h} A \epsilon^{d_{e}} \hat{p}\right] \boldsymbol{\nabla}_{\hat{\boldsymbol{x}}_{e}} \hat{p}-\boldsymbol{f}_{e}\left(\hat{\boldsymbol{x}}_{e}\right) \hat{p}\right\}
$$

where $\alpha_{h} A$ is the excluded-volume coefficient shown in Figure 4 As can be seen in the figure [or in the formulas for $\alpha_{h}$ [11)-(14)], the limit of $\alpha_{h} A$ as $h \rightarrow \infty$ is $\pi$ for the two-dimensional channel (NC2) and $4 \pi / 3$ for the three-dimensional cases (NC3) and (PP). Therefore, the limiting behaviour of (18) as $h \rightarrow \infty$ corresponds to replacing $\alpha_{h} A$ by $\alpha$, where the latter is given in the bulk equation (17). The last step to show that (17) is indeed the limiting model of (18) is to integrate (17) over the cross section to reduce it to a $d_{e}$-dimensional equation as (18). In other words, the limit $h \rightarrow \infty$ of the confined-domain model (18) should coincide with the limit $H \rightarrow 0$ of the bulk equation (17). Rescaling the confined dimensions by $\epsilon$ [cf. (5)] and integrating (17) over the cross-section, it is straightforward to arrive at the following equation for $\hat{p}=\epsilon^{k} p$ :

$$
\frac{\partial \hat{p}}{\partial t}\left(\hat{\boldsymbol{x}}_{e}, t\right)=\nabla_{\hat{\boldsymbol{x}}_{e}} \cdot\left\{\left[1+(N-1) \alpha \epsilon^{d_{e}} \hat{p}\right] \boldsymbol{\nabla}_{\hat{\boldsymbol{x}}_{e}} \hat{p}-\boldsymbol{f}_{e}\left(\hat{\boldsymbol{x}}_{e}\right) \hat{p}\right\},
$$

where we have used no-flux boundary conditions on the cross-section boundaries.

\subsection{Limit to single-file diffusion: $h \rightarrow 0$}

We now consider the limiting case $h \rightarrow 0$ in the narrow channel cases (NC2) and (NC3). From the plot of $\alpha_{h}$ in Figure 2 we have that $\lim _{h \rightarrow 0} \alpha_{h}=2$ in both cases. This is confirmed by taking the limit $h \rightarrow 0$ in (11) for (NC2) or (12) for (NC3). Thus the single-file limit of (10) is

$$
\frac{\partial \hat{p}_{e}}{\partial t}(\hat{x}, t)=\frac{\partial}{\partial \hat{x}}\left[\left[1+2(N-1) \epsilon \hat{p}_{e}\right] \frac{\partial \hat{p}_{e}}{\partial \hat{x}}-f_{1}(\hat{x}) \hat{p}_{e}\right] .
$$

We see that the effective diffusion coefficient for $N$ large (such that $N-1 \approx$ $N)$ is $D^{c}(c)=(1+2 c)$ with $c=N \epsilon \hat{p}_{e}$ being the particle concentration, which is consistent with that derived by Ackerson and Fleishman (1982) for a uniform particle concentration $c=N \epsilon$.

We now compare (20) with the classic one-dimensional model of diffusing hard rods. It is well known that the one-dimensional diffusion of finite-size particles 
can be mapped onto a point-particle problem (cf. Lizana and Ambiörnsson 2009). Using this trick, a fast diffusion equation for the evolution for the marginal density of $N$ rods of length $\epsilon$ under no external force $\left(f_{1} \equiv 0\right)$ and in the thermodynamic limit $(N \rightarrow \infty, L \rightarrow \infty, N / L \rightarrow \phi$ finite) is found in Rost (1984) (in French, see Bodnar and Velázquez 2005 for an explanation of Rost method in English), namely

$$
\frac{\partial \rho}{\partial t}(\hat{x}, t)=\frac{\partial}{\partial \hat{x}}\left(\frac{1}{(1-\epsilon \rho)^{2}} \frac{\partial \rho}{\partial \hat{x}}\right)
$$

where $\rho=N \hat{p}_{e}$ is the number density. Expanding the equation above in $\epsilon$ we obtain, to $\mathcal{O}(\epsilon)$,

$$
\frac{\partial \hat{p}_{e}}{\partial t}(\hat{x}, t)=\frac{\partial}{\partial \hat{x}}\left(\left(1+2 N \epsilon \hat{p}_{e}\right) \frac{\partial \hat{p}_{e}}{\partial \hat{x}}\right)
$$

which is in agreement with the large $N$ limit of (20). An alternative derivation of (20) using matched asymptotics on the original problem (without elimination of the hard-core parts) can be found in Bruna (2012). It differs from that in Rost (1984) in that it is valid for any $N$ and allows an external force field $\mathbf{f}$.

\subsection{Other limits}

From (14) we see that $\lim _{h \rightarrow 0} \alpha_{h}=\pi$ (see also Figure2), from which it is straightforward to show that the limit $h \rightarrow 0$ of (PP) corresponds to an unconfined twodimensional diffusion.

A final limit to consider concerns the generalisation of the three-dimensional channel (NC3) to a rectangular cross section $h \times m$. We have already seen above that this tends to the single-file diffusion model for $h=m \rightarrow 0$, and to an unconfined three-dimensional diffusion for $h, m \rightarrow \infty$. Now, keeping $h$ fixed, the extra parameter $m$ will allow us to interpolate between a two-dimensional narrow channel (NC2) of width $h$ as $m \rightarrow 0$ and two parallel plates a distance $h$ apart (PP) as $m \rightarrow \infty$. For $m \geq 1$ it can be shown that the rectangular counterpart of (12) reads:

$$
\alpha_{h m}=\frac{1}{h^{2} m^{2}}\left\{\frac{8}{15}+\Theta(h-1)\left[\frac{4 \pi}{3} h m-\frac{\pi}{2}(h+m)\right]+\Theta(1-h) s(h, m)\right\},
$$

with

$$
s(h, m)=\pi m h^{2}\left(1-\frac{h^{2}}{6}\right)-h \arcsin h+\frac{\sqrt{1-h^{2}}}{15}\left(2 h^{4}-9 h^{2}-8\right) .
$$

Now, in order to compare between the one-dimensional (NC3) model and the two-dimensional (PP) model, the relevant quantity is $m \alpha_{h m}$ (so that the onedimensional effective density $\hat{p}_{e}$ is mapped onto a two-dimensional plate of width $m)$. We find that

$$
\lim _{m \rightarrow \infty} m \alpha_{h m}^{(\mathrm{NC} 3)}=\alpha_{h}^{(\mathrm{PP})},
$$

as expected. 


\section{Model analysis}

4.1 Numerical analysis of time dependent solutions

In this section we compare solutions of our effective nonlinear diffusion equation for the (NC2) case with direct stochastic simulations, averaging over $2 \cdot 10^{4}$ stochastic realisations of the corresponding individual-based model (1). For the PDE, we use the method of lines with a standard second-order finite-difference discretisation of the spatial derivatives. For the coupled system of SDEs, we perform Monte Carlo (MC) simulations of the discretised version of (10) using the Euler-Maruyama method,

$$
\mathbf{X}_{i}(t+\Delta t)=\mathbf{X}_{i}(t)+\mathbf{f}\left(\mathbf{X}_{i}(t)\right) \Delta t+\sqrt{2 \Delta t} \boldsymbol{\xi}_{i},
$$

where $\boldsymbol{\xi}_{i}$ is a 2 -vector whose entries are independent normally distributed random variables with zero mean and unit variance. The reflective boundary conditions on $\partial \Omega$ implemented as in Erban et al (2007), namely, the distance that a particle has travelled outside the domain is reflected back into the domain. Care must be taken for very narrow channels to account for the possibility that a particle has travelled outside the domain by more than a width $h$. The particle-particle overlaps are implemented similarly: the difference $\epsilon-\left\|\mathbf{X}_{i}(t+\Delta t)-\mathbf{X}_{j}(t+\Delta t)\right\|$ corresponds to the distance that particles have penetrated each other illegally. Then we suppose that each particle has travelled the same illegal distance, and we separate them accordingly along the line joining the two particles' centres. This approach works well for low volume fractions, but may run into difficulty at high volume fractions when the separated particles may suffer further overlaps. In that case the algorithm in Scala et al (2007), an event-driven Brownian dynamics, becomes more suitable.
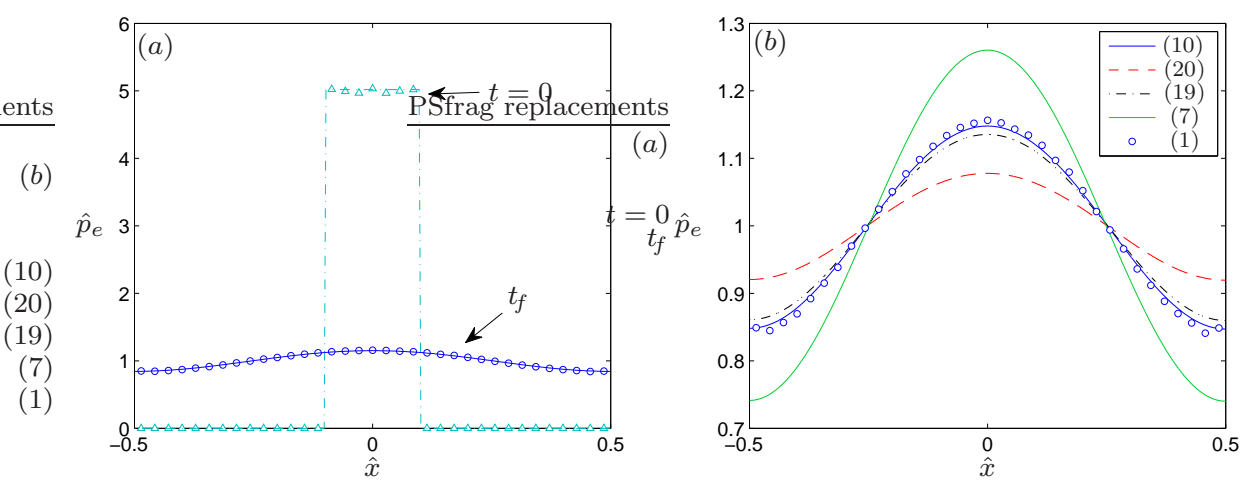

Figure 7 One-dimensional density $\hat{p}_{e}(\hat{x}, t)$ in a channel of width $\epsilon h$ with no-flux boundary conditions at $\hat{x}= \pm 0.5$ : solution of the continuum model 10 for finite-size particles versus individual-based model simulations of (1) and limiting continuum models. (a) Initial $(t=0)$ and final $\left(t_{f}=0.05\right)$ densities $\hat{p}_{e}$ (lines) and histograms (data points). (b) Final density and histogram, together with three limiting cases: point particles or standard linear diffusion (7), unconfined limit (19) and single-file limit 20). Parameters are $h=3, \epsilon=0.01$, and $N=30$, $\Delta t=10^{-5}$ 
Figures 7 and 8 show the numerical results at $t=0.05$ for $h=3, \epsilon=0.01$, $N=30, \mathbf{f}=0$ with no-flux and periodic boundary conditions at the channel ends, respectively. At initial time, the particles are uniformly distributed in a segment of length 0.2 [Figures 7(a) and 8(a) in cyan triangles and dash line]. The data points show the one-dimensional histogram obtained by averaging the $\mathrm{MC}$ results over the channel's cross section. To test the importance of the excluded-volume interactions and the confinement, we also compare with the corresponding solutions with point particles (equivalent to standard linear diffusion) and the limiting cases as $h \rightarrow \infty$ and $h \rightarrow 0$ of Subsections 3.1 and 3.2 respectively.
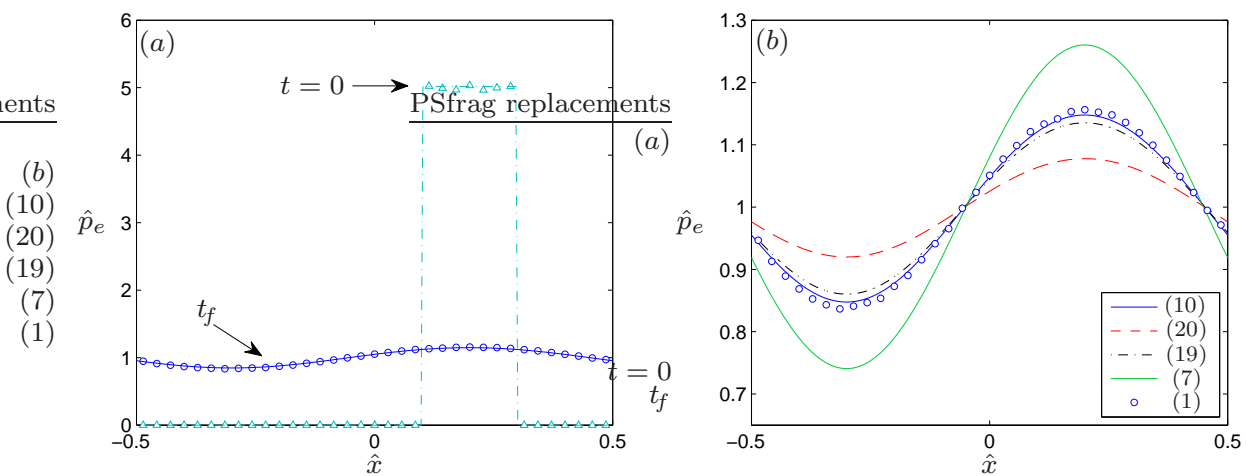

Figure 8 One-dimensional density $\hat{p}_{e}(\hat{x}, t)$ in a channel of width $\epsilon h$ with periodic boundary conditions at $\hat{x}= \pm 0.5$ : solution of the continuum model (10) for finite-size particles versus individual-based model simulations of (1) and limiting models (7), (19) and (20). Other details of the plots are given in the caption of Fig. 7 (Color figure online)

In both cases we see very good agreement between the stochastic average and the solution of the narrow-channel model $\hat{p}_{e}$, whilst there are noticeable differences between the three limiting models, namely the point particles, single-file and unconfined limits. In order to quantify the error committed by the limiting models, we note that, for the chosen parameters, the volume fraction is $\phi \approx 6 \%$ and the nonlinear coefficient in (16a) is $g_{h} \approx 4.6$. The corresponding nonlinear coefficient in the limiting cases is 0 for the point particles limit, 2 for the single-file limit and 4 for the unconfined limit. The difference between the narrow channel coefficient and these limiting values are consistent with the differences observed in the numerical solutions: while for $h=3$ the unconfined limit compares reasonably well with the stochastic simulations, the single-file and the point particles limits show more important differences. However, we note that our model gives the best agreement with the MC simulations results.

The results of Figures 7 and 8 suggest that, depending on the value of $h$, either the single-file or the unconfined limits will be more appropriate, while our narrowchannel model captures the whole range of $h$ from 0 to $\infty$. To investigate this further, in Figure 9 we examine how the narrow-channel model compares with three limiting cases [point particles (7), unconfined (19), and single-file (20)] for 
various values of the channel width $h$ while keeping the volume fraction $\phi$ fixed 2 It may seem counterintuitive that the limiting solutions appear to change with $h$ more than the narrow channel solution (solid blue line) does. However, we note that, although the three limiting models are independent of $h$, the unconfined model and the single-file model solutions (shown in dot-dash black and dash red lines respectively in Figure 9) do vary as $h$ increases because the value of $\epsilon$ is being changed in order to keep $\phi$ fixed. The narrow channel solution also changes (albeit less markedly) with $h$ as predicted by the coefficient $g_{h}$ (see Figure 5).
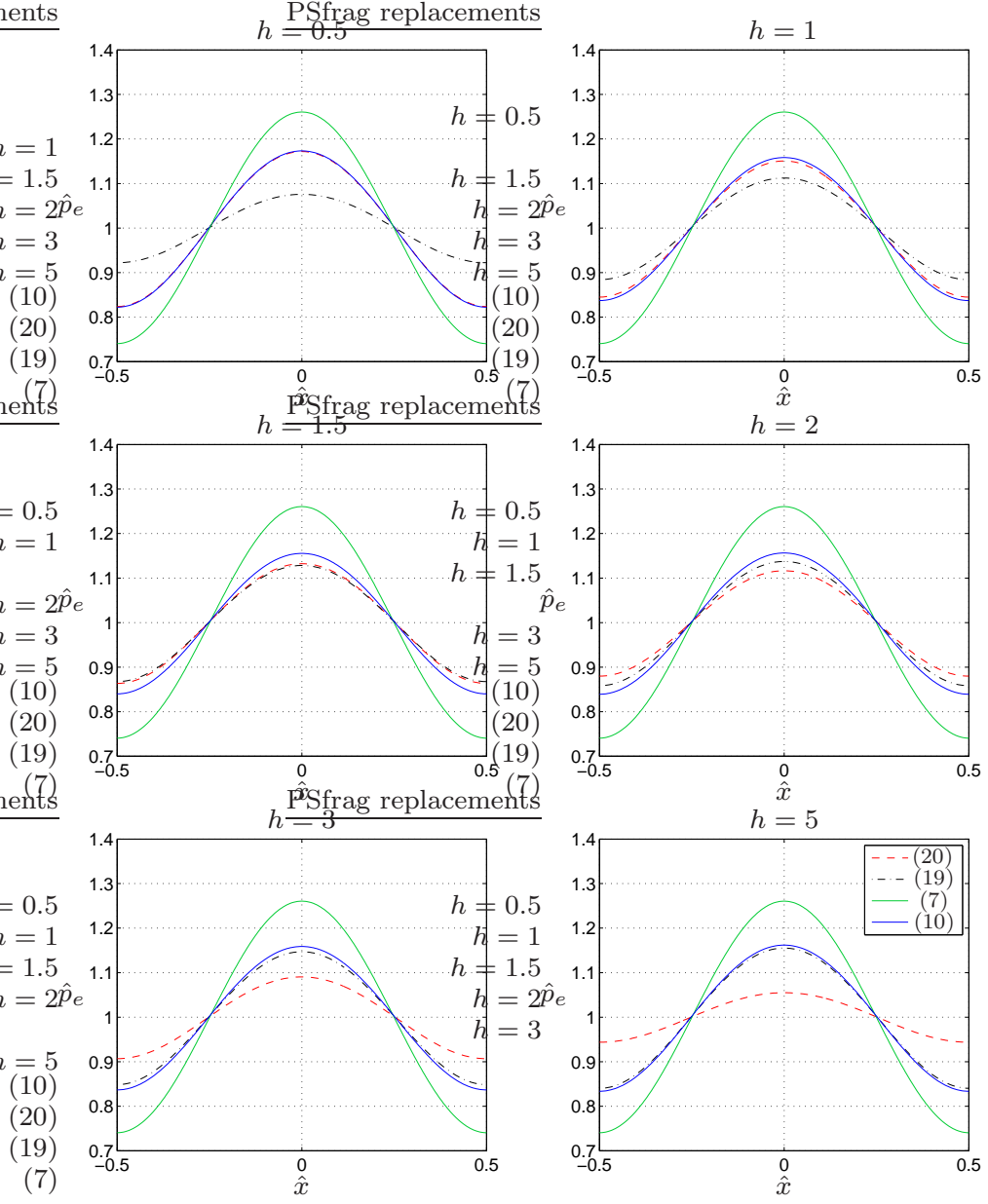

Figure 9 One-dimensional density $\hat{p}_{e}(\hat{x}, t)$ in a two-dimensional channel of width $\epsilon h$ with fixed volume fraction $\phi=0.05$ at time $t=0.05$. No-flux boundary conditions at $\hat{x}= \pm 0.5$ and uniformly initial conditions in $|\hat{x}| \leq 0.1$. Solution $\hat{p}_{e}$ of the narrow-channel equation (10) (solid blue), versus the single-file limit (20) (dash red line), the unconfined limit (19) (dot-dash black line, curve shown is $h \hat{p}$ ), and the point particles limit (7) (solid green line). (Color figure online)

\footnotetext{
${ }^{2}$ We keep the volume fraction $\phi=N \pi \epsilon / 4(h+1)$ fixed by varying $\epsilon$ as $h$ changes.
} 
As expected, for $h=0.5$ the narrow-channel solution agrees very well with the single-file limit, since in this case the cross-sectional space is not enough to let particles pass each other (see top right plot in Figure 3). In contrast, the unconfined case solution is far apart from the previous two, since the approximation that boundary effects are negligible is poor for $h=0.5$. As we increase $h$, we can observe how the single-file solution (in dash red) moves apart from the narrowchannel solution (in solid blue), while the unconfined case solution (in dot-dash black) becomes closer to the latter. When $h=5$ the narrow channel and unconfined model curves are nearly overlapping each other, indicating that at this channel width the boundary effects are almost negligible. These observations can also be made by looking at the graph of $g_{h}$ in Figure 5, in particular by considering the difference between $g_{h}$ at a given value of $h$ and the extreme values at $h=0, g_{0}=2$, and at $h=\infty, g_{\infty}=4$.

\subsection{Stationary solutions in periodic channels}

In this section we study the steady states of our continuum model and use the narrow-channel equation as an extension to the one-dimensional linear ratchet model for Brownian motors. For convenience, we focus on the one-dimensional equation (15) in terms of the coefficient $g_{h}$ for the narrow channel cases (NC2) and (NC3) only. As in the time-dependent case, the numerical solutions of the PDE are compared with corresponding stochastic simulations of the individualbased model (1). We suppose that the force along the channel axis $f_{1}(\hat{x})$ is the gradient of a potential $V(\hat{x})$, so that $f_{1}(\hat{x})=-V^{\prime}(\hat{x})$, where the prime indicates differentiation. Then (15) can be written as

$$
\frac{\partial \hat{p}_{e}}{\partial t}+\frac{\partial}{\partial \hat{x}}\left(\hat{p}_{e} u\right)=0, \quad \text { where } \quad u=-\frac{\partial}{\partial x}\left(\log \hat{p}_{e}+g_{h} \phi \hat{p}_{e}+V(\hat{x})\right) .
$$

The quantity $u$ can be interpreted as a flow down the gradient of the free energy $\mathcal{F}$ (Carrillo et al 2003) associated with (15); see Bruna (2012) for more details. The stationary solution of (25) with no-flux boundary conditions is obtained by minimising the free energy, which corresponds to solving

$$
\log \hat{p}_{e}+g_{h} \phi \hat{p}_{e}+V(\hat{x})=C,
$$

with the constant $C$ determined by the normalisation condition on $\hat{p}_{e}$. For the application to ratchet systems, we are interested in periodic solutions of (25) with a (constant) flux $J_{0} \equiv \hat{p}_{e} u 3^{3}$

The one-dimensional Fokker-Planck equation (7) for point particles with $f_{1}(\hat{x})=$ $-V^{\prime}(\hat{x})$ can be used to model directed particle transport under ratchet potentials (i.e. potentials spatially asymmetric with respect to their maxima Slater et al 1997). These potentials may describe a periodic asymmetric free-energy substrate in the case for molecular motors through microtubules Kolomeisky and Fisher 2007) or steric interactions in the case of polyelectrolytes (Slater et al 1997). In particular, theoretical approaches to molecular motors such as kinesin have focused on either a one-dimensional continuum model such as (7) (thus ignoring the

\footnotetext{
3 For periodic boundary data, $J_{0}$ is an extra degree of freedom determined by imposing periodicity.
} 
interactions between different motors and the other dimensions) or on stochastic simple-exclusion models on a lattice (Kolomeisky and Fisher 2007). More generally, in these applications the modelling has focused on the form of the ratchet potential in order to make the model more realistic, instead on the possible interactions between the particles involved in the transport. In the remainder of this section we will explore the effects that interactions (specifically, excluded-volume interactions, but this could be extended to other types of interactions) can have on such models. To this end, we consider a specific ratchet model with a ratchet potential consisting of a periodic part plus a constant external force or tilt. We use the tilted Smoluchowski-Feynman potential

$$
V\left(\hat{x}, F_{0}\right)=\sin (2 \pi \hat{x})+0.25 \sin (4 \pi \hat{x})-F_{0} \hat{x} .
$$

Two plots of $V(\hat{x})$ for different values of the tilt $F_{0}$ are shown in Figure 10. It can be shown that in the long-time limit the sign of the particle current (or net motion) agrees with the sign of the tilt $F_{0}$ and that, in the absence of tilt $\left(F_{0}=0\right)$ there is no net motion (Reimann 2002). An interesting feature of this model is that the relationship between the tilt $F_{0}$ and the flux $J_{0}$ is nonlinear (see thick black line in Figure 12).
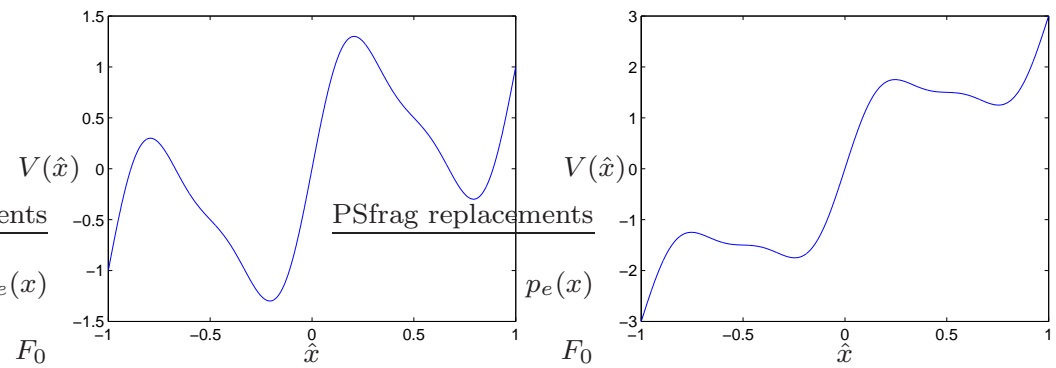

Figure 10 Tilted Smoluchowski-Feynman ratchet potential $V\left(\hat{x}, F_{0}\right)$ in Eq. 26] for $F_{0}=-1$ (left) and $F_{0}=-3$ (right).

Next we examine how the $F_{0}$ vs. $J_{0}$ relationship changes when nonlinear effects (due to the finite-size of particles and confinement) are included in the model. To this end, we solve for the stationary states of (25) when $V(\hat{x})$ is given by (26) for various tilts $F_{0}$ and compute the stationary flux $J_{0}$ of the resulting solution. For each tilt $F_{0}$, we must find the flux $J_{0}$ and the stationary solution $\hat{p}_{e}(\hat{x})$ such that the periodic and normalisation conditions are fulfilled:

$$
\begin{aligned}
\left(1+g_{h} \phi \hat{p}_{e}\right) \hat{p}_{e}^{\prime}+V\left(\hat{x}, F_{0}\right) \hat{p}_{e} & =-J_{0}, \\
\hat{p}_{e}(-1 / 2) & =\hat{p}_{e}(1 / 2), \\
\int_{-1 / 2}^{1 / 2} \hat{p}_{e} \mathrm{~d} \hat{x} & =1 .
\end{aligned}
$$

We solve this problem numerically using Chebfun (Trefethen and others 2011) in MATLAB. Solutions of (27) for an increasing value of the constant force $F_{0}$ (varying from -6 to +6 ) are shown in Figure 11. The left panel corresponds to point particles $\left(g_{h}=0\right)$, while the right panel corresponds to finite-size particles 

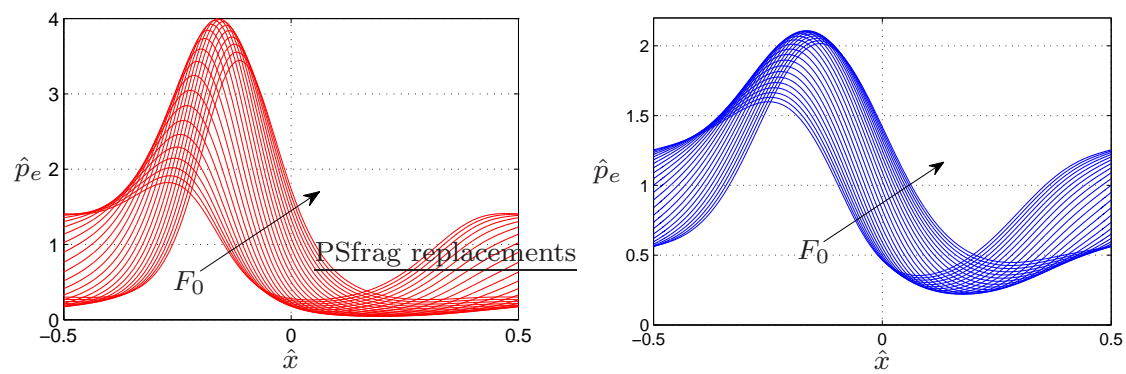

Figure 11 Stationary solution $\hat{p}_{e}(\hat{x})$ under the tilted Smoluchowski-Feynman potential 26 for various values of $F_{0}$. Stationary solutions of 25 for point particles, $g_{h}=0$ (left) and for finite-size particles with $g_{h} \phi=0.6$ (right).

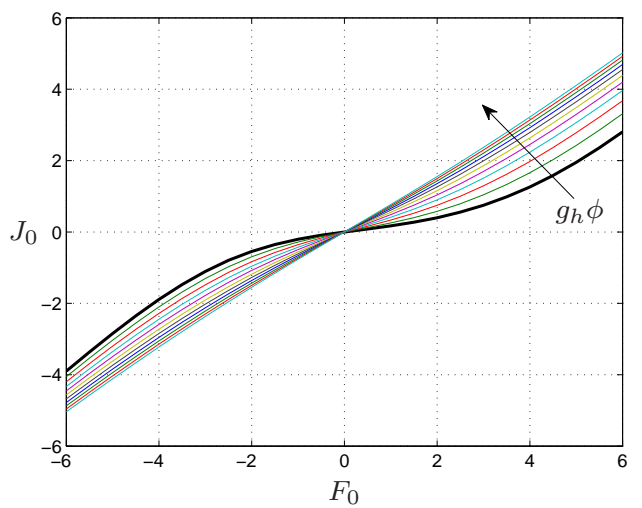

Figure 12 Steady-state flux $J_{0}$ versus tilt $F_{0}$ from solving $(25)$ for increasing values of $g_{h} \phi$ (from 0 to 1). The coloured lines correspond to finite-size particles $\left(g_{h}>0\right)$. The thick black line corresponds to point particles $\left(g_{h}=0\right)$ as shown in Reimann 2002, Figure 2.4).

with $g_{h} \phi=0.6$. The diagram of the resulting steady flux $J_{0}$ versus the tilt $F_{0}$ is shown in Figure 12 for increasing values of $g_{h} \phi$. We observe that the relationship is nonlinear for point particles $\left(g_{h}=0\right.$, thick black line), but it appears to become linear as excluded-volume effects get larger (i.e. as $g_{h} \phi$ increases). This is physically reasonable, since point particles get easily trapped in the wells of the potential, even if these wells are relatively small. In contrast, it is easier for finite-size particles to escape, as they may not all fit in the potential well and the nonlinear diffusion makes the potential barrier easier to overcome.

In Figure 13 we compare the stationary solutions $\hat{p}_{e}$ for point particles $\left(g_{h}=0\right)$ and finite-size particles (with an excluded-volume coefficient of $g_{h}=0.15$ ) in two tilting scenarios: for $F_{0}=-6$ and for $F_{0}=2.5$. We observe that while the solutions are almost overlapping for a tilt of $F_{0}=-6$, they are considerably different for $F_{0}=2.5$. This is because for $F_{0}=-6$ the potential $V$ is so tilted that it ceases to have a local minimum within each period. As a result, the "advantage" of finitesize particles that could more easily overcome the local minima in the potential is lost. 


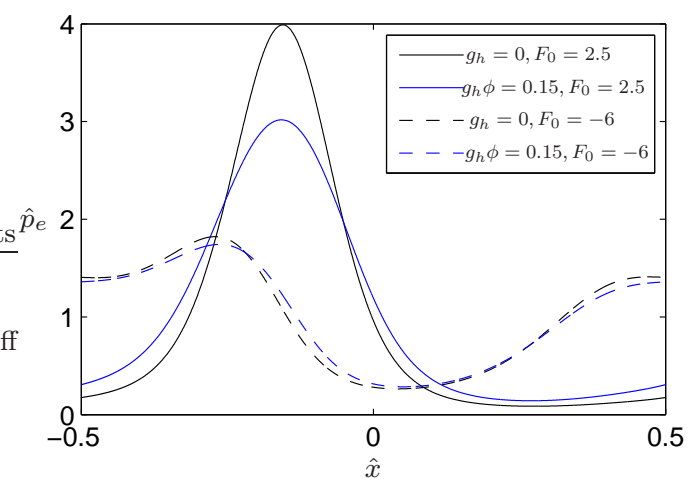

Figure 13 Stationary solution $\hat{p}_{e}\left(\hat{x}_{1}\right)$ under the potential (26). Solutions of 25 for $g_{h}=0$ and $g_{h}=0.15$, and $F_{0}=-6$ and $F_{0}=2.5$.

We conclude this section by comparing the equilibrium solutions of the continuum model with the corresponding stochastic simulations of the discrete model. We use the Metropolis-Hastings algorithm (Chib and Greenberg 1995) to sample from the stationary density of the full-particle system, and compare the resulting histogram (averaged over the cross section) with the stationary solutions $\hat{p}_{e}$ of (25). We consider the (NC2) case for which the coefficient $g_{h}$ is maximised for a fixed volume fraction $\phi$, that is, a channel of width $h^{*}=1.47$. The other parameters used in the simulations are $\epsilon=10^{-3}, N=133$ and $F_{0}=2.5$. Figure [14 dis-

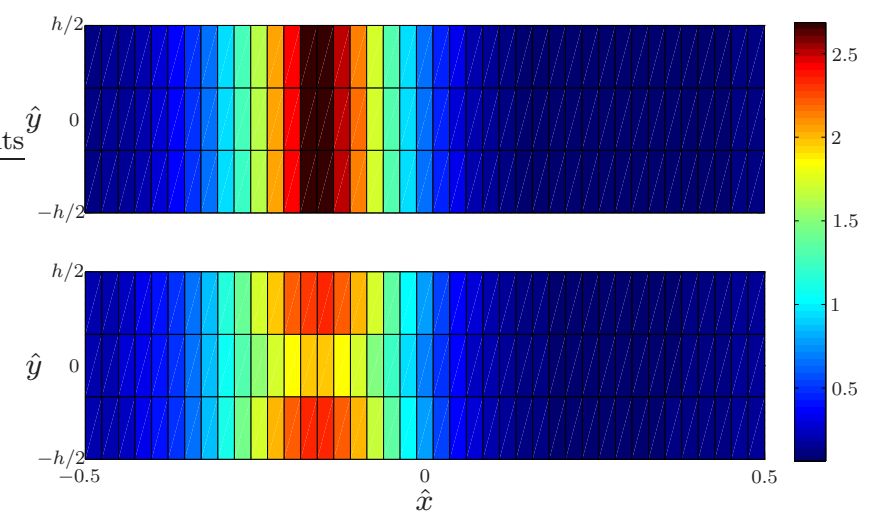

Figure 14 Histogram of the stationary density $\hat{p}(\hat{\mathbf{x}})$ under the potential $V\left(\hat{x}, F_{0}\right)$ in 26 for point particles (top plot) and finite-size particles (bottom plot). Parameters are $F_{0}=2.5$, $h=1.47, \epsilon=10^{-3}, N=133$. Histograms computed by $10^{7} \mathrm{MH}$ steps.

plays the histograms after $10^{7}$ steps of the $\mathrm{MH}$ algorithm. The histogram for point particles (upper plot in Figure 14) does not vary in the cross-sectional direction, as expected. In contrast, the histogram for finite-size particles does display some variation in the $\hat{y}$-direction: more particles want to be near the boundaries than 
in the centre of the channel. This is because a hard-disk particle on the boundary excludes only half of the area that would exclude in the centre of the domain (recall that the channel of width $h$ is the domain available to the particles centres, not the physical domain), and this is entropically favourable. In our derivation of equation (10) this variation is taken into account but integrated out to obtain the one-dimensional equation. Accounting for the variation in $\hat{y}$ is not the main objective of this paper but this could be done either by numerically solving the two-dimensional equation (48) or evaluating higher order terms in the expansion of section A.2.

We plot the theoretical predictions by solving (25) for both point and finitesize particles alongside their respective simulation counterparts, and observe an excellent agreement in both cases. We examine the importance of taking into account the actual width of the channel, which in this example is only $h=1.47$, by solving the analogous stationary problem for the single-file model (20). We plot the result (dot-dash black line in Figure 15) and observe that the single-file model overestimates the excluded-volume effects, as demonstrated by the flatter density profile.

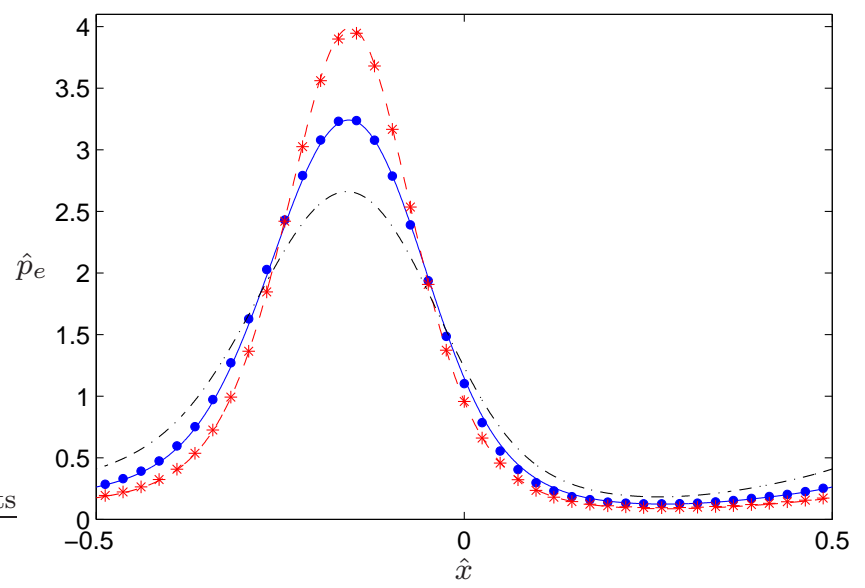

Figure 15 One-dimensional stationary density $\hat{p}_{e}(\hat{x})$ under the potential $V\left(\hat{x}, F_{0}\right)$ in (26). Solutions of 25 for point particles $\left(g_{h}=0\right.$, dash red line) and finite-size particles $\left(g_{h}=0.1\right.$, solid blue line), with $F_{0}=2.5, N=133, h=1.47, \epsilon=10^{-3}$. Cross-sectional averages of the histograms in Figure 14 for point particles (red asterisks) and finite-size particles (blue circles). Stationary solution of the single-file equation (20) is shown in a dot-dash black line.

\section{Discussion}

In this paper, we have presented a derivation of a continuum model for the diffusion of finite-size particles in a confined domain whose dimensions are comparable to the particle dimensions. We have given the model explicitly for three confined geometries, namely a two- and three-dimensional narrow channel of square crosssection and Hele-Shaw cell (two close parallel plates), but also indicated how the 
model derivation for more general geometries can be done. The resulting continuum model is a nonlinear drift-diffusion equation for the one-particle probability density or for the population volume concentration, with the nonlinear term depending on the excluded-volume created by the particles as well as the confinement parameter (in the cases we have studied, the confinement parameter is the channel width or the separation between plates). This equation is defined in the effective domain, a lower-dimensional domain for the unconfined dimensions only, exploiting the fact that equilibration in the confined dimensions is relatively fast and most dynamics occur along the unconfined ones. For example, in the case of a two- or threedimensional channel the resulting continuum model is a one-dimensional equation along the axial direction.

The derivation of the final continuum model involved two key steps. First, as in our previous works (Bruna and Chapman 2012a, b), we used the method of matched asymptotic expansions to reduce the high-dimensional Fokker-Planck equation associated with the individual-based description of the system to a lowdimensional Fokker-Planck equation for the one-particle density function. Second, we exploited the confined geometry of the domain to perform a further reduction and integrate out the confined dimensions of the problem. While in our previous works the particle-particle-wall interactions were a higher-order correction, and thus were neglected, in this work they were taken into account. In other words, while in an unconfined domain such three-body interactions simply create a boundary layer near the domain walls, in the situations considered in the present work this boundary layer extends across the cross section and must be solved accurately.

The model has two interesting features. First, we found that, for a given volume fraction, there exists an optimal ratio $h$ between confinement and particle size such that the excluded-volume effects are maximised. This means, for example, that one can design a lab-on-a-chip device to achieve a maximal collective diffusion coefficient. Second, the model is capable of describing the whole range of confinement levels and interpolating between confinement extremes, that is, between extreme confinement $(h=0)$ when particles cannot pass each other to unconfined diffusion $(h=\infty)$. We have examined the limiting models corresponding to each of the three geometries we have studied, and found that our model agrees with them. For example, in the narrow-channel cases, the extreme confinement corresponds to a single-file diffusion model (Rost 1984) while the other limit is an unconfined two or three-dimensional diffusion model (Bruna and Chapman $2012 \mathrm{~b}$ ). This is a useful analytical tool to predict the error that is being committed by using the limiting models, that is, either ignoring the fact that particles can (just) pass each other and using the purely one-dimensional single-file equation, or neglecting confinement conditions and boundary layers.

In order to assess the validity of our model predictions, we have compared the numerical results of our continuum model for the two-dimensional narrow channel to the results from the corresponding individual-based model as well as the limiting continuum models of point particles, single-file diffusion and unconfined diffusion. We have observed excellent agreement between the narrow channel model and the stochastic simulations of the discrete model, and confirmed the interpolating properties of the model between confinement extremes. Finally, we have discussed a case study involving the diffusion under a ratchet potential, used for example to describe the transport of molecular motors through microtubules. We have examined the effects that interactions between particles and confinement conditions can 
have in the analysis of the problem. For example, we have found that an increase on excluded-volume effects causes the particle transport to be less sensitive to the tilting of the ratchet potential.

There are several directions along which one can extend this model. Firstly, it would be interesting to allow multiple types of particles (labelled blues and reds, say) by combining the analysis in Bruna and Chapman (2012a) with the present work. A challenging aspect of this problem is that the resulting model of two species in a narrow channel must account for the fact that as the channel becomes single file the order of particles is fixed, and since the two types of particles are distinguishable the order of particles matters. In other words, we expect a qualitative difference in the model as $h$ crosses the value of one: for $h>1$, even if all the red particles were initially to the left of the blue particles, we expect the two populations to mix together (that is, the jump in the initial density profiles will spread); in contrast, for $h<1$, if the two populations are segregated initially, they will stay like that for all times, and we expect to see this refelected in the population-level densities. In connection with this issue is the fact that the self-diffusion of a particle is not defined in one-dimensional systems (Ackerson and Fleishman 1982). Another extension is to allow channels of varying cross section, that is, to extend the Fick-Jacobs model (9) to the case of finite-size particles. A simplification of this problem (ignoring the particle-particle interactions) has been considered by Riefler et al (2010). A related problem would be to match a narrow channel with two bulk domains in each end; such a geometry could have important applications in the area of ion channels.

Acknowledgements This publication was based on work supported in part by Award No. KUK-C1-013-04, made by King Abdullah University of Science and Technology (KAUST). MB acknowledges financial support from EPSRC. We are grateful to the organisers of the workshop "Stochastic Modelling of Reaction-Diffusion Processes in Biology", which has led to this Special Issue.

\section{A Derivation of the narrow-channel equation (10) for the (NC2) case}

This section is devoted to the derivation of (10) in the two-dimensional channel (NC2) case. The derivation of the three-dimensional cases (NC3) and (PP) or other (simple) geometries follows similarly (see Subsection A.3 for an outline of the conditions/calculations to be carried out).

A.1 Transformation $\mathcal{T}_{1}$ : Reduction from the individual- to the population-level

Our starting point is the Fokker-Planck equation for $N$ hard-disc particles (2a), defined in the high-dimensional (configuration) space $\Omega_{\epsilon}^{N} \subset \mathbb{R}^{2 N}$. Recall that the configuration space has holes that correspond to illegal configurations (with particles' overlaps) on which the no-flux boundary conditions $2 \mathrm{~b}$ hold. The aim of this subsection is to derive the corresponding Fokker-Planck equation for the one-particle density $p\left(\mathbf{x}_{1}, t\right)=\int P(\vec{x}, t) \mathrm{d} \mathbf{x}_{2} \ldots \mathbf{x}_{N}$, where $P(\vec{x}, t)$ is the joint probability density of the $N$ particles. We first note that the integration of (2) over $\mathbf{x}_{2}, \ldots, \mathbf{x}_{N}$ results in integrals over contact surfaces on which $P$ must be evaluated (Bruna and Chapman 2012b). When the particle volume is small, the dominant contributions to these contact integrals correspond to two-particle interactions, so that we can set $N=2$ and then extend the result to $N$ arbitrary in a straightforward manner. However, in contrast with the unconfined case studied in Bruna and Chapman (2012b), under confinement conditions 
the particle-particle-wall interactions (three-body) are not negligible and must be taken into account. For two particles at positions $\mathbf{x}_{1}$ and $\mathbf{x}_{2}, 2$ reads

$$
\begin{aligned}
\frac{\partial P}{\partial t}\left(\mathbf{x}_{1}, \mathbf{x}_{2}, t\right) & =\boldsymbol{\nabla}_{\mathbf{x}_{1}} \cdot\left[\boldsymbol{\nabla}_{\mathbf{x}_{1}} P-\mathbf{f}\left(\mathbf{x}_{1}\right) P\right]+\boldsymbol{\nabla}_{\mathbf{x}_{2}} \cdot\left[\boldsymbol{\nabla}_{\mathbf{x}_{2}} P-\mathbf{f}\left(\mathbf{x}_{2}\right) P\right] \quad \text { in } \quad \Omega_{\epsilon}^{2}, \\
0 & =\left[\boldsymbol{\nabla}_{\mathbf{x}_{1}} P-\mathbf{f}\left(\mathbf{x}_{1}\right) P\right] \cdot \hat{\mathbf{n}}_{1}+\left[\boldsymbol{\nabla}_{\mathbf{x}_{2}} P-\mathbf{f}\left(\mathbf{x}_{2}\right) P\right] \cdot \hat{\mathbf{n}}_{2},
\end{aligned}
$$

on $\mathbf{x}_{i} \in \partial \Omega$ and $\left\|\mathbf{x}_{1}-\mathbf{x}_{2}\right\|=\epsilon$. Here $\hat{\mathbf{n}}_{i}=\mathbf{n}_{i} /\left\|\mathbf{n}_{i}\right\|$, where $\mathbf{n}_{i}$ is the component of the normal vector $\vec{n}$ corresponding to the $i$ th particle, $\vec{n}=\left(\mathbf{n}_{1}, \mathbf{n}_{2}\right)$. We note that $\hat{\mathbf{n}}_{1}=0$ on $\mathbf{x}_{2} \in \partial \Omega$, and that $\hat{\mathbf{n}}_{1}=-\hat{\mathbf{n}}_{2}$ on $\left\|\mathbf{x}_{1}-\mathbf{x}_{2}\right\|=\epsilon$.

\section{A.1.1 From $N$ particles to 1 particle}

We denote by $\Omega\left(\mathbf{x}_{1}\right)=\Omega \backslash B_{\epsilon}\left(\mathbf{x}_{1}\right)$ the region available to the centre of particle 2 when particle 1 is at $\mathbf{x}_{1}$. Note that when the distance between $\mathbf{x}_{1}$ and $\partial \Omega$ is less than $\epsilon$ the area $\left|\Omega\left(\mathbf{x}_{1}\right)\right|$ increases (see Figure 16$)$ because the area $\mathcal{U}\left(\mathbf{x}_{1}\right)=B_{\epsilon}\left(\mathbf{x}_{1}\right) \cap \Omega$ excluded by particle 1 changes with $\mathbf{x}_{1}$. The points on which the two particles are in contact are given by the collision boundary

$$
\mathcal{C}_{\mathbf{x}_{1}}=\left\{\mathbf{x}_{2} \in \Omega \text { s.t. }\left\|\mathbf{x}_{2}-\mathbf{x}_{1}\right\|=\epsilon\right\} .
$$

Integrating Eq. 28a over $\Omega\left(\mathbf{x}_{1}\right)$ using the Reynolds transport theorem (on the moving boundary $\mathcal{C}_{\mathbf{x}_{1}}$ ), the divergence theorem, and the boundary condition 28b yields

$$
\frac{\partial p}{\partial t}\left(\mathbf{x}_{1}, t\right)=\nabla_{\mathbf{x}_{1}} \cdot\left[\nabla_{\mathbf{x}_{1}} p-\mathbf{f}\left(\mathbf{x}_{1}\right) p\right]+\int_{\mathcal{C}_{\mathbf{x}_{1}}}-\left(\nabla_{\mathbf{x}_{1}} P+\nabla_{\mathbf{x}_{2}} P\right) \cdot \mathbf{n}_{2} \mathrm{~d} S_{2} .
$$

We denote the collision integral above by $\mathcal{I}$. If we now consider the case of $N$ particles we would obtain a collision integral for each pair, so that after some particle relabelling the corresponding equation is

$$
\frac{\partial p}{\partial t}\left(\mathbf{x}_{1}, t\right)=\nabla_{\mathbf{x}_{1}} \cdot\left[\nabla_{\mathbf{x}_{1}} p-\mathbf{f}\left(\mathbf{x}_{1}\right) p\right]+(N-1) \int_{\mathcal{C}_{\mathbf{x}_{1}}}-\left(\nabla_{\mathbf{x}_{1}} P+\nabla_{\mathbf{x}_{2}} P\right) \cdot \mathbf{n}_{2} \mathrm{~d} S_{2} .
$$

Equation (31) is halfway through transformation $\mathcal{T}_{1}$ (cf. Figure 1), since the first half of the equation depends only on $\mathbf{x}_{\mathbf{1}}$ while the integral $\mathcal{I}$ still depends on the two-particle density $P$ near the collision surface $\mathcal{C}_{\mathbf{x}_{1}}$.

At this stage, it is common to use a closure approximation such as $P\left(\mathbf{x}_{1}, \mathbf{x}_{2}, t\right)=p\left(\mathbf{x}_{1}, t\right) p\left(\mathbf{x}_{2}, t\right)$ to evaluate $\mathcal{I}$ and obtained a closed equation for $p$ (Rubinstein and Keller 1989). However, the pairwise particle interaction-and therefore the correlation between their positions-is exactly localised near the collision surface $\mathcal{C}_{\mathbf{x}_{1}}$. Instead, in the next section we will use an alternative method based on matched asymptotic expansions to evaluate $\mathcal{I}$ systematically (Bruna and Chapman 2012b).

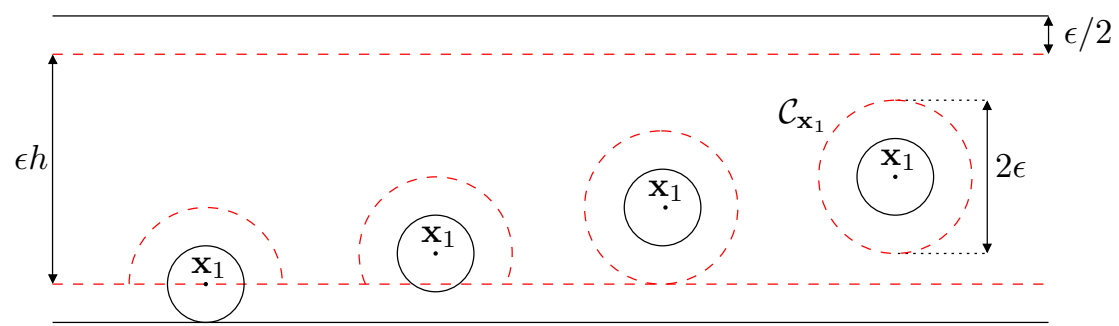

Figure 16 Sketch of the original channel geometry (solid black lines) and the effective configuration space for the centre of a second circular particle, given by the boundary function $\partial \Omega\left(\mathbf{x}_{1}\right)$ (dash red lines) which depends on the position of the first particle $\mathbf{x}_{1}$. The collision boundary function $\mathcal{C}_{\mathbf{x}_{1}}$ forms part of the effective boundary $\partial \Omega\left(\mathbf{x}_{1}\right)$. 


\section{A.1.2 Matched asymptotic expansions}

We suppose that when two particles are far apart $\left(\left|x_{1}-x_{2}\right| \gg 1\right)$ they are independent (at leading order), whereas when they are close to each other $\left(\left|x_{1}-x_{2}\right| \sim \epsilon\right)$ they are correlated. We denote these two regions of the configuration space $\Omega_{\epsilon}^{2}$ the outer region and the inner region, respectively. We use the $x$-coordinate to distinguish between the two regions because the inner region spans the channel's cross section. Importantly this implies that the outer region is disconnected.

Outer region In the outer region we consider the change to the narrow-domain variables and define $\hat{P}\left(\hat{\mathbf{x}}_{1}, \hat{\mathbf{x}}_{2}, t\right)=\epsilon^{2} P\left(\mathbf{x}_{1}, \mathbf{x}_{2}, t\right)$. This scaling is consistent with that introduced for the one-particle density in Section 2.2 and is such that $P$ and $\hat{P}$ each integrate to one in their respective domains $\Omega_{\epsilon}^{2}$ and the narrow-domain variable equivalent $\omega_{\epsilon}^{2}$. Then (28a) becomes

$$
\begin{aligned}
\epsilon^{2} \frac{\partial \hat{P}}{\partial t}\left(\hat{\mathbf{x}}_{1}, \hat{\mathbf{x}}_{2}, t\right)= & \frac{\partial}{\partial \hat{y}_{1}}\left(\frac{\partial \hat{P}}{\partial \hat{y}_{1}}-\epsilon f_{2}\left(\hat{x}_{1}, \epsilon \hat{y}_{1}\right) \hat{P}\right)+\frac{\partial}{\partial \hat{y}_{2}}\left(\frac{\partial \hat{P}}{\partial \hat{y}_{2}}-\epsilon f_{2}\left(\hat{x}_{2}, \epsilon \hat{y}_{2}\right) \hat{P}\right) \\
& +\epsilon^{2} \frac{\partial}{\partial \hat{x}_{1}}\left(\frac{\partial \hat{P}}{\partial \hat{x}_{1}}-f_{1}\left(\hat{x}_{1}, \epsilon \hat{y}_{1}\right) \hat{P}\right)+\epsilon^{2} \frac{\partial}{\partial \hat{x}_{2}}\left(\frac{\partial \hat{P}}{\partial \hat{x}_{2}}-f_{1}\left(\hat{x}_{2}, \epsilon \hat{y}_{2}\right) \hat{P}\right),
\end{aligned}
$$

for $\left(\hat{\mathbf{x}}_{1}, \hat{\mathbf{x}}_{2}\right) \in \omega_{\epsilon}^{2}$, with

$$
\begin{aligned}
\frac{\partial \hat{P}}{\partial \hat{x}_{i}}-f_{1}\left(\hat{x}_{i}, \epsilon \hat{y}_{i}\right) \hat{P}=0 & \text { on } & \hat{x}_{i}= \pm \frac{1}{2} \\
\frac{\partial \hat{P}}{\partial \hat{y}_{i}}-\epsilon f_{2}\left(\hat{x}_{i}, \epsilon \hat{y}_{i}\right) \hat{P}=0 & \text { on } & \hat{y}_{i}= \pm \frac{h}{2},
\end{aligned}
$$

for $i=1,2$. The boundary condition on the collision line $\mathcal{C}_{\mathbf{x}_{1}}$ disappears for $\left|\hat{x}_{1}-\hat{x}_{2}\right|>\epsilon$, so that it is "invisible" to the outer region

In the outer region, we define $P_{\text {out }}\left(\hat{\mathbf{x}}_{1}, \hat{\mathbf{x}}_{2}, t\right)=\hat{P}\left(\hat{\mathbf{x}}_{1}, \hat{\mathbf{x}}_{2}, t\right)$ and look for an asymptotic solution to (32) by expanding $P_{\text {out }}$ in powers of $\epsilon$. We find at leading order that $P_{\text {out }}$ must be independent of the vertical coordinates $\hat{y}_{1}$ and $\hat{y}_{2}$. By independence in the outer region we suppose that the leading-order solution is separable, so that it is of the form $q\left(\hat{x}_{1}, t\right) q\left(\hat{x}_{2}, t\right)$ for some function $q$. Solving for the next two orders in $\epsilon$ we find that the solution in the outer region is, to $\mathcal{O}\left(\epsilon^{2}\right)$,

$$
\begin{aligned}
P_{\text {out }}\left(\hat{\mathbf{x}}_{1}, \hat{\mathbf{x}}_{2}, t\right) & =q\left(\hat{x}_{1}, t\right) q\left(\hat{x}_{2}, t\right) \\
+\epsilon & \left\{\left(\hat{x}_{1}, t\right) q\left(\hat{x}_{2}, t\right)\left[\hat{y}_{1} f_{2}\left(\hat{x}_{1}, 0\right)+\hat{y}_{2} f_{2}\left(\hat{x}_{2}, 0\right)\right]+\Upsilon_{1}\left(\hat{x}_{1}, \hat{x}_{2}, t\right)\right\} \\
+ & \epsilon^{2}\left\{\frac{1}{2}\left[\hat{y}_{1}^{2} \frac{\partial f_{2}}{\partial y}\left(\hat{x}_{1}, 0\right)+\hat{y}_{2}^{2} \frac{\partial f_{2}}{\partial y}\left(\hat{x}_{2}, 0\right)+\left(\hat{y}_{1} f_{2}\left(\hat{x}_{1}, 0\right)+\hat{y}_{2} f_{2}\left(\hat{x}_{2}, 0\right)\right)^{2}\right]\right. \\
& \left.\quad+\Upsilon_{1}\left(\hat{x}_{1}, \hat{x}_{2}, t\right)\left[\hat{y}_{1} f_{2}\left(\hat{x}_{1}, 0\right)+\hat{y}_{2} f_{2}\left(\hat{x}_{2}, 0\right)\right]+\Upsilon_{2}\left(\hat{x}_{1}, \hat{x}_{2}, t\right)\right\}
\end{aligned}
$$

where the $\Upsilon_{i}\left(\hat{x}_{1}, \hat{x}_{2}, t\right)$ are arbitrary functions of integration, determined by solvability conditions at higher order. Note that the invariance of $P$ with respect to a switch of particle labels means that in the outer region both particles have the same density function $q$. From the solvability condition on the $O\left(\epsilon^{2}\right)$ terms above we obtain the following equation for $q$ :

$$
\frac{\partial q}{\partial t}(\hat{x}, t)=\frac{\partial}{\partial \hat{x}}\left(\frac{\partial q}{\partial \hat{x}}-f_{1}(\hat{x}, 0) q\right) .
$$

Inner region In the inner region we introduce the inner variables

$$
x_{1}=\tilde{x}_{1}, \quad y_{1}=\epsilon \tilde{y}_{1}, \quad x_{2}=\tilde{x}_{1}+\epsilon \tilde{x}, \quad y_{2}=\epsilon \tilde{y}_{2},
$$

and define $\tilde{P}\left(\tilde{\mathbf{x}}_{1}, \tilde{\mathbf{x}}_{2}, t\right)=\epsilon^{2} P\left(\mathbf{x}_{1}, \mathbf{x}_{2}, t\right)$. The contact boundary $\mathcal{C}_{\mathbf{x}_{1}} \underline{29}$ becomes

$$
\tilde{\mathcal{C}}_{\tilde{y}_{1}}=\left\{\left(\tilde{x}, \tilde{y}_{2}\right) \in \mathbb{R} \times[-h / 2, h / 2] \text { s.t. } \tilde{x}^{2}+\left(\tilde{y}_{2}-\tilde{y}_{1}\right)^{2}=1\right\},
$$

\footnotetext{
${ }^{4}$ To see this, we write (29) in terms of the narrow variables. It becomes $\mathcal{C}_{\hat{\mathbf{x}}_{1}}=\left\{\hat{\mathbf{x}}_{2} \in \omega\right.$ : $\left.\left(\hat{x}_{2}-\hat{x}_{1}\right)^{2}+\epsilon^{2}\left(\hat{y}_{2}-\hat{y}_{1}\right)^{2}=\epsilon^{2}\right\}$.
} 
and problem (28) is transformed to

$$
\begin{aligned}
\epsilon^{2} \frac{\partial \tilde{P}}{\partial t}\left(\tilde{\mathbf{x}}_{1}, \tilde{\mathbf{x}}_{2}, t\right)= & 2 \frac{\partial^{2} \tilde{P}}{\partial \tilde{x}^{2}}+\frac{\partial^{2} \tilde{P}}{\partial \tilde{y}_{1}^{2}}+\frac{\partial^{2} \tilde{P}}{\partial \tilde{y}_{2}^{2}}-2 \epsilon \frac{\partial^{2} \tilde{P}}{\partial \tilde{x}_{1} \partial \tilde{x}}-\epsilon \frac{\partial}{\partial \tilde{y}_{2}}\left[f_{2}\left(\tilde{x}_{1}+\epsilon \tilde{x}, \epsilon \tilde{y}\right) \tilde{P}\right] \\
& +\epsilon \frac{\partial}{\partial \tilde{x}}\left\{\left[f_{1}\left(\tilde{x}_{1}, \epsilon \tilde{y}_{1}\right)-f_{1}\left(\tilde{x}_{1}+\epsilon \tilde{x}, \epsilon \tilde{y}_{2}\right)\right] \tilde{P}\right\} \\
& -\epsilon \frac{\partial}{\partial \tilde{y}_{1}}\left[f_{2}\left(\tilde{x}_{1}, \epsilon \tilde{y}_{1}\right) \tilde{P}\right]+\epsilon^{2} \frac{\partial^{2} \tilde{P}}{\partial \tilde{x}_{1}^{2}}-\epsilon^{2} \frac{\partial}{\partial \tilde{x}_{1}}\left[f_{1}\left(\tilde{x}_{1}, \epsilon \tilde{y}_{1}\right) \tilde{P}\right]
\end{aligned}
$$

with

$$
\begin{aligned}
2 \tilde{x} \frac{\partial \tilde{P}}{\partial \tilde{x}}+\left(\tilde{y}_{2}-\tilde{y}_{1}\right)\left(\frac{\partial \tilde{P}}{\partial \tilde{y}_{2}}-\frac{\partial \tilde{P}}{\partial \tilde{y}_{1}}\right)= & \epsilon \tilde{x} \frac{\partial \tilde{P}}{\partial \tilde{x}_{1}}+\epsilon \tilde{x}\left[f_{1}\left(\tilde{x}_{1}+\epsilon \tilde{x}, \epsilon \tilde{y}_{2}\right)-f_{1}\left(\tilde{x}_{1}, \epsilon \tilde{y}_{1}\right)\right] \tilde{P} \\
& +\epsilon\left(\tilde{y}_{2}-\tilde{y}_{1}\right)\left[f_{2}\left(\tilde{x}_{1}+\epsilon \tilde{x}, \epsilon \tilde{y}_{2}\right)-f_{2}\left(\tilde{x}_{1}, \epsilon \tilde{y}_{1}\right)\right] \tilde{P}
\end{aligned}
$$

on $\tilde{\mathcal{C}}_{\tilde{y}_{1}}$ and

$$
\begin{array}{lll}
\frac{\partial \tilde{P}}{\partial \tilde{y}_{1}}=\epsilon f_{2}\left(\tilde{x}_{1}, \epsilon \tilde{y}_{1}\right) \tilde{P} & \text { on } & \tilde{y}_{1}= \pm \frac{h}{2} \\
\frac{\partial \tilde{P}}{\partial \tilde{y}_{2}}=\epsilon f_{2}\left(\tilde{x}_{1}+\epsilon \tilde{x}, \epsilon \tilde{y}_{2}\right) \tilde{P} & \text { on } & \tilde{y}_{2}= \pm \frac{h}{2}
\end{array}
$$

In addition to $37 \mathrm{~b}-37 \mathrm{~d}$, the inner solution must match with the outer as $\tilde{x} \rightarrow \pm \infty$. Expanding the outer solution in terms of the inner variables, which corresponds to replacing $\hat{x}_{1}=\tilde{x}_{1}$, $\hat{y}_{1}=\tilde{y}_{1}, \hat{x}_{2}=\tilde{x}_{1}+\epsilon \tilde{x}$ and $\hat{y}_{2}=\tilde{y}_{2}$ in 33 , and subsequently expanding in powers of $\epsilon$, we obtain the following matching condition:

$$
\begin{aligned}
\tilde{P} \sim q^{2}+ & \epsilon\left[\tilde{x} q \frac{\partial q}{\partial \tilde{x}_{1}}+\left(\tilde{y}_{1}+\tilde{y}_{2}\right) f_{2} q^{2}\left(\tilde{x}_{1}\right)+\Upsilon_{1}\left(\tilde{x}_{1}, \tilde{x}_{1}\right)\right] \\
+\epsilon^{2} & {\left[\frac{\tilde{x}^{2}}{2} q \frac{\partial^{2} q}{\partial \tilde{x}_{1}^{2}}+\tilde{x}\left(\tilde{y}_{1}+\tilde{y}_{2}\right) f_{2} q q_{\tilde{x}_{1}}+\tilde{x} \tilde{y}_{2} \frac{\partial f_{2}}{\partial x} q^{2}+\tilde{x} \frac{\partial \Upsilon_{1}}{\partial \hat{x}_{2}}\left(\tilde{x}_{1}, \tilde{x}_{1}\right)\right.} \\
& +\frac{1}{2}\left(\left(\tilde{y}_{1}^{2}+\tilde{y}_{2}^{2}\right) \frac{\partial f_{2}}{\partial y}+\left(\tilde{y}_{1}+\tilde{y}_{2}\right)^{2} f_{2}^{2}\right) q^{2}+\left(\tilde{y}_{1}+\tilde{y}_{2}\right) f_{2} \Upsilon_{1}\left(\tilde{x}_{1}, \tilde{x}_{1}\right) \\
& \left.+\Upsilon_{2}\left(\tilde{x}_{1}, \tilde{x}_{1}\right)\right]+\cdots \quad \text { as } \quad \tilde{x} \rightarrow \pm \infty
\end{aligned}
$$

where $q \equiv q\left(\tilde{x}_{1}, t\right)$. Expanding $\tilde{P}$ in powers of $\epsilon, \tilde{P} \sim \tilde{P}^{(0)}+\epsilon \tilde{P}^{(1)}+\epsilon^{2} \tilde{P}^{(2)}+\cdots$, we find that the leading- and first-order solutions of [37] are

$$
\begin{aligned}
& \tilde{P}^{(0)}=q^{2}\left(\tilde{x}_{1}\right), \\
& \tilde{P}^{(1)}=\tilde{x} q\left(\tilde{x}_{1}\right) \frac{\partial q}{\partial \tilde{x}_{1}}\left(\tilde{x}_{1}\right)+\left(\tilde{y}_{1}+\tilde{y}_{2}\right) f_{2}\left(\tilde{x}_{1}, 0\right) q^{2}\left(\tilde{x}_{1}\right)+\Upsilon_{1}\left(\tilde{x}_{1}, \tilde{x}_{1}\right) .
\end{aligned}
$$

Unlike in the bulk or unconfined problem (Bruna and Chapman 2012a b), the narrow-channel problem requires computing the second-order inner solution. The solution procedure is rather cumbersome and is omitted here. It involves a further change of variable $\tilde{x}=\sqrt{2} \tilde{s}$ to turn the problem into a Poisson problem, and solving two sub-problems numerically using the commercial finite-element solver COMSOL Multiphysics 4.3. The second-order solution of (37) is (see Appendix C.1 in Bruna 2012 for full details)

$$
\begin{aligned}
\tilde{P}^{(2)}= & \frac{1}{2} \tilde{x}^{2} q \frac{\partial^{2} q}{\partial \tilde{x}_{1}^{2}}+\frac{1}{2}\left(\tilde{y}_{1}^{2}+\tilde{y}_{2}^{2}\right) q^{2}\left(\frac{\partial f_{2}}{\partial y}+f_{2}^{2}\right)+\tilde{y}_{1} \tilde{y}_{2} f_{2}^{2} q^{2} \\
& +\tilde{x}\left(\tilde{y}_{1} f_{2} q \frac{\partial q}{\partial \tilde{x}_{1}}+\tilde{y}_{2} q \frac{\partial\left(f_{2} q\right)}{\partial \tilde{x}_{1}}\right)+\left(\frac{\partial f_{1}}{\partial y}-\frac{\partial f_{2}}{\partial x}\right) q^{2} \tilde{Q}_{2}\left(\tilde{x}, \tilde{y}_{1}, \tilde{y}_{2}\right) \\
& +\sqrt{2}\left[q \frac{\partial^{2} q}{\partial \tilde{x}_{1}^{2}}-\left(\frac{\partial q}{\partial \tilde{x}_{1}}\right)^{2}-\frac{\partial f_{1}}{\partial x} q^{2}\right] \tilde{Q}_{1}\left(\tilde{x}, \tilde{y}_{1}, \tilde{y}_{2}\right)+\Upsilon_{2}\left(\tilde{x}_{1}, \tilde{x}_{1}\right)
\end{aligned}
$$

with $\tilde{Q}_{i}\left(\tilde{x}, \tilde{y}_{1}, \tilde{y}_{2}\right)=\tilde{v}_{i}\left(\tilde{x} / \sqrt{2}, \tilde{y}_{1}, \tilde{y}_{2}\right)$, where $\tilde{v}_{1}\left(\tilde{s}, \tilde{y}_{1}, \tilde{y}_{2}\right)$ and $\tilde{v}_{2}\left(\tilde{s}, \tilde{y}_{1}, \tilde{y}_{2}\right)$ are given by (numerical solutions of)

$$
\begin{aligned}
& \widetilde{\nabla}^{2} \tilde{v}_{1}=0, \\
& \tilde{\boldsymbol{\nabla}} \tilde{v}_{1} \cdot \tilde{\boldsymbol{\nu}}=\tilde{s}^{2} \quad \text { on } \quad \tilde{\mathcal{D}}_{\tilde{y}_{1}} \text {, } \\
& \tilde{\nabla} \tilde{v}_{1} \cdot \tilde{\boldsymbol{\nu}}=0 \quad \text { on } \quad \tilde{y}_{i}= \pm \frac{h}{2} \text {, } \\
& \tilde{v}_{1} \sim D_{1}|\tilde{s}| \quad \text { as } \quad \tilde{s} \rightarrow \pm \infty,
\end{aligned}
$$


and

$$
\begin{array}{rlrlrl}
\tilde{\nabla}^{2} \tilde{v}_{2} & =0, & & \\
\widetilde{\boldsymbol{\nabla}} \tilde{v}_{2} \cdot \tilde{\boldsymbol{\nu}} & =\tilde{s}\left(\tilde{y}_{1}-\tilde{y}_{2}\right), & & \text { on } & & \tilde{\mathcal{D}}_{\tilde{y}_{1}}, \\
\widetilde{\boldsymbol{\nabla}} \tilde{v}_{2} \cdot \tilde{\boldsymbol{\nu}} & =0, & & \text { on } & & \tilde{y}_{i}= \pm \frac{h}{2}, \\
\tilde{v}_{2} & \sim 0, & & \text { as } & & \tilde{s} \rightarrow \pm \infty .
\end{array}
$$

Here $\widetilde{\nabla}$ stands for the gradient operator with respect to the position vector $\left(\tilde{s}, \tilde{y}_{1}, \tilde{y}_{2}\right), \tilde{\mathcal{D}}_{\tilde{y}_{1}}$ is the transformed collision boundary $\tilde{\mathcal{C}}_{\tilde{y}_{1}}(36)$, and $\tilde{\boldsymbol{\nu}}$ is the outward unit normal on this mapped boundary, $\tilde{\boldsymbol{\nu}}=-\frac{\sqrt{2}}{2}\left(2 \tilde{s}, \tilde{y}_{1}-\tilde{y}_{2}, \tilde{y}_{2}-\tilde{y}_{1}\right)$. Finally, the constant field $D_{1}$ at infinity for $\tilde{v}_{1}$ is related to the integration function from the outer $\Upsilon_{1}$,

$$
D_{1}=\frac{\lim _{\hat{x}_{2} \rightarrow \hat{x}_{1}} \frac{\partial \Upsilon_{1}}{\partial \hat{x}_{2}}\left(\hat{x}_{1}, \hat{x}_{2}\right)}{\left[q \frac{\partial^{2} q}{\partial \tilde{x}_{1}^{2}}-\left(\frac{\partial q}{\partial \tilde{x}_{1}}\right)^{2}-\frac{\partial f_{1}}{\partial x} q^{2}\right]} .
$$

Out of the derivation we also find that $\Upsilon_{1}\left(\hat{x}_{1}, \hat{x}_{2}\right) \equiv \Upsilon_{1}\left(\left|\hat{x}_{1}-\hat{x}_{2}\right|\right)$ with $\Upsilon_{1}(x)$ differentiable satisfying $\Upsilon_{1}(0)=0$. In contrast, the contribution from the other outer function $\Upsilon_{2}$ is left unknown (it could be determined by matching higher order terms), but we are able to ignore it as it has a zero contribution to the collision integral $\mathcal{I}$ (as we will see in the next section). We note that, in (40), $q, f_{1}$ and $f_{2}$ are functions of the "outer" variable $\tilde{x}_{1}$ only, namely, $q=q\left(\tilde{x}_{1}, t\right)$ and $f_{i}=f_{i}\left(\tilde{x}_{1}, 0\right)$.

Combining (38), (39) and (40) we have the solution to the inner problem (37) up to $\mathcal{O}\left(\epsilon^{2}\right)$.

\section{A.1.3 Collision integral}

Now we go back to Eq. (31) and use the asymptotic solution of the previous subsection to turn it into an equation for $p\left(\mathbf{x}_{1}, t\right)$ only thus completing transformation $\mathcal{T}_{1}$. Note that, since the integral $\mathcal{I}$ is over the collision boundary $\mathcal{C}_{\mathbf{x}_{1}}$, it lives in the inner region and we must use $\tilde{P}$ to evaluate it.

In terms of the inner variables, $\mathcal{I}$ is

$$
\mathcal{I}=\epsilon^{-2} \int_{\tilde{\mathcal{C}}_{\tilde{y}_{1}}}\left[\left(\tilde{y}_{2}-\tilde{y}_{1}\right)\left(\frac{\partial \tilde{P}}{\partial \tilde{y}_{1}}+\frac{\partial \tilde{P}}{\partial \tilde{y}_{2}}\right)+\epsilon \tilde{x} \frac{\partial \tilde{P}}{\partial \tilde{x}_{1}}\right] \mathrm{d} \tilde{l},
$$

where $\tilde{\mathcal{C}}_{\tilde{y}_{1}}$ is given in (36) and d $\tilde{l}$ is the line integral along this curve (for $\tilde{y}_{1}$ fixed, see Figure 17). Depending on the channel width $h$ (relative to one, which is the radius of $\tilde{\mathcal{C}}_{\tilde{y}_{1}}$ ), the integration is over the whole circle or a part of it. Introducing the distances $l_{1}=\max \left(-h / 2-\tilde{y}_{1},-1\right)$ and $l_{2}=\min \left(h / 2-\tilde{y}_{1}, 1\right)$, the angles at contact with the lower and upper channel walls are $\theta_{1}=\arcsin l_{1}$ and $\theta_{2}=\arcsin l_{2}$, respectively. (These are equal to $\pm \pi / 2$ for no contact.)

Writing $\mathcal{I}=\epsilon^{-2}\left(\mathcal{I}^{(0)}+\epsilon \mathcal{I}^{(1)}+\epsilon^{2} \mathcal{I}^{(2)}+\cdots\right)$ and using 43), we find that

$$
\begin{aligned}
\mathcal{I}^{(0)}= & 0, \\
\mathcal{I}^{(1)}= & 2 f_{2} q^{2} \mu_{0}\left(h, \tilde{y}_{1}\right), \\
\mathcal{I}^{(2)}= & 2 q^{2}\left(\frac{\partial f_{2}}{\partial y}+2 f_{2}^{2}\right) \tilde{y}_{1} \mu_{0}\left(h, \tilde{y}_{1}\right)+\left(2 q \frac{\partial^{2} q}{\partial \tilde{x}_{1}^{2}}-q^{2} \frac{\partial f_{1}}{\partial x}\right) \mu_{1}\left(h, \tilde{y}_{1}\right) \\
& +q^{2}\left(\frac{\partial f_{2}}{\partial y}+2 f_{2}^{2}\right) \mu_{2}\left(h, \tilde{y}_{1}\right)+2\left(\frac{\partial f_{1}}{\partial y}-\frac{\partial f_{2}}{\partial x}\right) q^{2} \mathcal{J}\left[\tilde{Q}_{2}\right]\left(h, \tilde{y}_{1}\right) \\
& +2 \sqrt{2}\left[q \frac{\partial^{2} q}{\partial \tilde{x}_{1}^{2}}-\left(\frac{\partial q}{\partial \tilde{x}_{1}}\right)^{2}-\frac{\partial f_{1}}{\partial x} q^{2}\right] \mathcal{J}\left[\tilde{Q}_{1}\right]\left(h, \tilde{y}_{1}\right),
\end{aligned}
$$




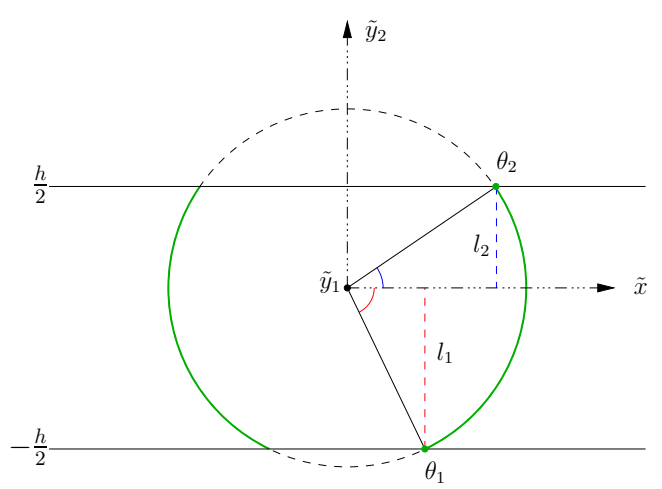

Figure 17 Domain of integration $\tilde{\mathcal{C}}_{\tilde{y}_{1}}$ (solid green line), distances $l_{1}$ and $l_{2}$ between the vertical coordinate of the first particle and the lower and upper channel walls and corresponding angles $\theta_{1}$ and $\theta_{2}$.

where $f_{i}=f_{i}\left(\tilde{x}_{1}, 0\right)$,

$$
\begin{aligned}
& \mu_{0}\left(h, \tilde{y}_{1}\right)=\int_{\tilde{\mathcal{C}}_{\tilde{y}_{1}}}\left(\tilde{y}_{2}-\tilde{y}_{1}\right) \mathrm{d} \tilde{l}=2\left(\sqrt{1-l_{1}^{2}}-\sqrt{1-l_{2}^{2}}\right), \\
& \mu_{1}\left(h, \tilde{y}_{1}\right)=\int_{\tilde{\mathcal{C}}_{\tilde{y}_{1}}} \tilde{x}^{2} \mathrm{~d} \tilde{l}=l_{2} \sqrt{1-l_{2}^{2}}-l_{1} \sqrt{1-l_{1}^{2}}+\arcsin l_{2}-\arcsin l_{1}, \\
& \mu_{2}\left(h, \tilde{y}_{1}\right)=\int_{\tilde{\mathcal{C}}_{\tilde{y}_{1}}}\left(\tilde{y}_{2}-\tilde{y}_{1}\right)^{2} \mathrm{~d} \tilde{l}=l_{1} \sqrt{1-l_{1}^{2}}-l_{2} \sqrt{1-l_{2}^{2}}-\arcsin l_{1}+\arcsin l_{2},
\end{aligned}
$$

and $\mathcal{J}$ is the integral operator $\mathcal{J}[Q]\left(h, \tilde{y}_{1}\right)=\int_{\tilde{\mathcal{C}}_{\tilde{y}_{1}}}\left[Q_{\tilde{y}_{2}}\left(\tilde{y}_{2}-\tilde{y}_{1}\right)+Q_{\tilde{x}} \tilde{x}\right] \mathrm{d} \tilde{l}$. The terms $\mathcal{J}\left[\tilde{Q}_{1}\right]$ and $\mathcal{J}\left[\tilde{Q}_{2}\right]$ are evaluated numerically with COMSOL (see Appendix C.2 in Bruna 2012 for more details).

\section{A.1.4 Population-level Fokker-Planck equation}

Combining (45) and (46) we obtain the first two terms of the asymptotic expansion for $\mathcal{I}$, which depends on both the channel width $h$ and the elevation of the first particle $\tilde{y}_{1}$ but is independent of the position of the second particle. Thus we can drop the first particle label (the subindex 1) for clarity of notation. Inserting this expansion into (31), we obtain an equation for the first particle

$$
\frac{\partial p}{\partial t}(\mathbf{x}, t)=\boldsymbol{\nabla}_{\mathbf{x}} \cdot\left[\boldsymbol{\nabla}_{\mathbf{x}} p-\mathbf{f}(\mathbf{x}) p\right]+(N-1)\left(\epsilon^{-1} \mathcal{I}^{(1)}+\mathcal{I}^{(2)}\right) \quad \text { in } \quad \Omega,
$$

which involves the marginal density $p(\mathbf{x}, t)$, the outer density $q(\hat{x}, t)$ and the channel width $h$. This concludes the transformation $\mathcal{T}_{1}$ from $N$ particles to one particle (see Figure 1).

\section{A.2 Transformation $\mathcal{T}_{2}$ : Reduction of the number of geometric dimensions}

Following a similar procedure to that for point particles in Section 2.2 we will reduce (48) into a one-dimensional effective equation along the axial direction. First, integrating 28b over $\Omega(\mathbf{x})$ for $\mathbf{x} \in \partial \Omega$, we obtain the following no-flux boundary condition:

$$
\left[\nabla_{\mathbf{x}} p-\mathbf{f}(\mathbf{x}) p\right] \cdot \hat{\mathbf{n}}=0 \quad \text { on } \quad \partial \Omega .
$$


Analogously to the point-particles case, we use the narrow-domain variables (5) and define $\hat{p}(\hat{\mathbf{x}}, t)=\epsilon p(\mathbf{x}, t)$. With this rescaling, Eqs. 480 and become

$$
\begin{aligned}
\epsilon^{2} \frac{\partial \hat{p}}{\partial t}(\hat{\mathbf{x}}, t) & =\frac{\partial}{\partial \hat{y}}\left(\frac{\partial \hat{p}}{\partial \hat{y}}-\epsilon f_{2} \hat{p}\right)+\epsilon^{2} \frac{\partial}{\partial \hat{x}}\left(\frac{\partial \hat{p}}{\partial \hat{x}}-f_{1} \hat{p}\right)+(N-1) \epsilon^{2}\left(\mathcal{I}^{(1)}+\epsilon \mathcal{I}^{(2)}\right), \\
\frac{\partial \hat{p}}{\partial \hat{x}} & =f_{1} \hat{p} \quad \text { on } \quad \hat{x}= \pm \frac{1}{2} \\
\frac{\partial \hat{p}}{\partial \hat{y}} & =\epsilon f_{2} \hat{p} \quad \text { on } \quad \hat{y}= \pm \frac{h}{2}
\end{aligned}
$$

where $f_{i} \equiv f_{i}(\hat{x}, \epsilon \hat{y})$. There is no need to expand the integral terms $\mathcal{I}^{(i)}$ in terms of the narrow-domain variables since these are written in terms of the inner variables (35), which coincide with the narrow-domain variable for expressions independent of the second particle's coordinates.

Expanding $\hat{p}$ in powers of $\epsilon$ and solving (50) gives, at leading order, that $\hat{p}$ is independent of $\hat{y}$. As before, we introduce the effective one-dimensional densities as $\hat{p}_{e}^{(i)}=\int_{-h / 2}^{h / 2} \hat{p}^{(i)} \mathrm{d} \hat{y}$. Thus we have that $\hat{p}_{e}^{(0)} \equiv h \hat{p}_{e}^{(0)}$. At the next order,

$$
\hat{p}^{(1)}(\hat{\mathbf{x}}, t)=f_{2}(\hat{x}, 0) \hat{p}^{(0)}(\hat{x}, t) \hat{y}+\hat{p}_{e}^{(1)}(\hat{x}, t) / h .
$$

For clarity of notation, in the remaining of this section we write $f_{i}(\hat{x}, 0) \equiv f_{i}$. Integrating the second order of (50a) over the channel's cross section and using (50c), gives

$$
\frac{\partial \hat{p}_{e}^{(0)}}{\partial t}(\hat{x}, t)=\frac{\partial}{\partial \hat{x}}\left(\frac{\partial \hat{p}_{e}^{(0)}}{\partial \hat{x}}-f_{1} \hat{p}_{e}^{(0)}\right),
$$

where we have used that $\int_{-h / 2}^{h / 2} \mathcal{I}^{(1)} \mathrm{d} \hat{y}=0$ [see Eqs. 450 and 47a ]. Note that this equation coincides with the effective equation for point particles (7). It is at the next order that the finite-size effects appear.

Repeating the same procedure of integrating with respect to $\hat{y}$ the $\mathcal{O}\left(\epsilon^{3}\right)$ of (50) and using 50c to eliminate $\hat{p}^{(3)}$ yields the following solvability condition

$$
\frac{\partial \hat{p}_{e}^{(1)}}{\partial t}(\hat{x}, t)-\frac{\partial}{\partial \hat{x}}\left(\frac{\partial \hat{p}_{e}^{(1)}}{\partial \hat{x}}-f_{1} \hat{p}_{e}^{(1)}\right)=(N-1) \int_{-h / 2}^{h / 2} \mathcal{I}^{(2)} \mathrm{d} \hat{y} .
$$

Using (46) and 477, the cross-section integral of $\mathcal{I}^{(2)}$ is

$$
\begin{aligned}
\int_{-h / 2}^{h / 2} \mathcal{I}^{(2)} \mathrm{d} \hat{y}= & \left(2 q \frac{\partial^{2} q}{\partial \tilde{x}_{1}^{2}}-q^{2} \frac{\partial f_{1}}{\partial x}\right) M_{1}(h)+2\left(\frac{\partial f_{1}}{\partial y}-\frac{\partial f_{2}}{\partial x}\right) q^{2} \mathcal{M}\left[\tilde{Q}_{2}\right] \\
& +2 \sqrt{2}\left[q \frac{\partial^{2} q}{\partial \tilde{x}_{1}^{2}}-\left(\frac{\partial q}{\partial \tilde{x}_{1}}\right)^{2}-\frac{\partial f_{1}}{\partial x} q^{2}\right] \mathcal{M}\left[\tilde{Q}_{1}\right]
\end{aligned}
$$

where $M_{1}(h)=\int_{-h / 2}^{h / 2} \mu_{1}(h, \hat{y}) \mathrm{d} \hat{y}$ reads

$$
M_{1}(h)=\pi h-\frac{4}{3}+\Theta(1-h)\left[\frac{2}{3}\left(2+h^{2}\right) \sqrt{1-h^{2}}-2 h \arccos (h)\right],
$$

where $\Theta(x)$ is the Heaviside step function, and $\mathcal{M}[Q](h)=\int_{-h / 2}^{h / 2} \mathcal{J}[Q](h, \hat{y}) \mathrm{d} \hat{y}$. Although $\tilde{Q}_{1}$ and $\tilde{Q}_{2}$ are only solved numerically, using information from their respective problems (41) and (42) one can deduce analytical expressions for their integrals $\mathcal{M}\left[\tilde{Q}_{i}\right]$, namely that $\mathcal{M}\left[\tilde{Q}_{1}\right]=-M_{1}(h) /(2 \sqrt{2})$ and $\mathcal{M}\left[\tilde{Q}_{2}\right]=0$ (see Appendix C.3 in Bruna 2012). Using this, we find that

$$
\int_{-h / 2}^{h / 2} \mathcal{I}^{(2)} \mathrm{d} \hat{y}=\frac{\partial}{\partial \tilde{x}_{1}}\left(q \frac{\partial q}{\partial \tilde{x}_{1}}\right) M_{1}(h) .
$$


Because $q$ is independent of the inner variables $\left(\tilde{x}, \tilde{y}_{1}, \tilde{y}_{2}\right)$, we can write $q\left(\tilde{x}_{1}, t\right)=q_{e}(\hat{x}, t) / h$. Moreover, the normalisation condition on $\hat{P}$ gives that $q_{e}(\hat{x}, t)=\hat{p}_{e}(\hat{x}, t)+\mathcal{O}(\epsilon)$. Therefore the right-hand side of (56) becomes $\frac{\partial}{\partial \hat{x}}\left(\hat{p}_{e} \frac{\partial \hat{p}_{e}}{\partial \hat{x}}\right) / h^{2}$.

Combining (52), (53) and (56) yields

$$
\frac{\partial \hat{p}_{e}}{\partial t}(\hat{x}, t)=\frac{\partial}{\partial \hat{x}}\left\{\left[1+(N-1) \epsilon \frac{M_{1}(h)}{h^{2}} \hat{p}_{e}\right] \frac{\partial \hat{p}_{e}}{\partial \hat{x}}-f_{1}(\hat{x}, 0) \hat{p}_{e}\right\},
$$

which coincides with the effective equation (10) for a two-dimensional narrow-channel after writing $\alpha_{h} \equiv M_{1}(h) / h^{2}$; see (11).

\section{A.3 Outline of steps for other geometries}

In this section we indicate the key steps to derive the effective continuum Fokker-Planck equation (10) for a general geometry, and in particular for the three-dimensional cases (NC3) and (PP) presented in Section 2.3 First, we note below relevant definitions that change with the problem dimension $d$ and the number of confined dimensions $k$ (recall that $d_{e}=d-k$ ):

(1) Identify the number of confined and effective dimensions: the original position vector is split into two components, $\mathbf{x}=\left(\boldsymbol{x}_{e}, \boldsymbol{x}_{c}\right)$ (see Table 1). For example, for (NC3) $\boldsymbol{x}_{e}=x$ and $\boldsymbol{x}_{c}=(y, z)$, while for $(\mathrm{PP}) \boldsymbol{x}_{e}=(x, y)$ and $\boldsymbol{x}_{c}=z$.

\begin{tabular}{l|c|c|c|} 
& Dimension & Variables & Domain \\
Original problem & $d$ & $\mathbf{x}$ & $\Omega$ \\
Confined space & $k$ & $\boldsymbol{x}_{c}$ & $\Omega_{c}$ \\
Effective problem & $d_{e}$ & $\boldsymbol{x}_{e}$ & $\Omega_{e}$
\end{tabular}

Table 1 Dimension and variables in each of the spaces and subspaces.

(2) Determine the confinement parameter(s): Next we must choose an scaling for the confined dimensions relative to the unconfined ones. In all cases considered here we made that simple by saying that all confined dimensions are of length $H$ relative to the unconfined ones, but there could be of different lengths too, such as the narrow channel of rectangular cross section mentioned briefly in Subsec. 3.3 Suppose that the confinement dimensions are $\boldsymbol{H}=\left(H_{1}, \ldots, H_{k}\right)=\mathcal{O}(\epsilon)$. Then the vector of confinement parameters is given by $\boldsymbol{h}=\left(h_{1}, \ldots, h_{k}\right)$, with $h_{i}=H_{i} / \epsilon$.

Note that we are assuming that a confined dimension is always of order $\epsilon$ (the particle's diameter). However, this could also be generalised and introduce an intermediate scaling (between $\epsilon$ and one)

(3) Narrow-domain variables transformation: the generalisation of the change of variables (5) is

$$
\boldsymbol{x}_{e}=\hat{\boldsymbol{x}}_{e}, \quad \boldsymbol{x}_{c}=\epsilon \hat{\boldsymbol{x}}_{c} .
$$

We write $\hat{\mathbf{x}}=\left(\hat{\boldsymbol{x}}_{e}, \hat{\boldsymbol{x}}_{c}\right)$. The one-particle and two-particle densities in the rescaled domain are respectively defined as

$$
\hat{p}\left(\hat{\mathbf{x}}_{1}, t\right)=\epsilon^{k} p\left(\mathbf{x}_{1}, t\right), \quad \hat{P}\left(\hat{\mathbf{x}}_{1}, \hat{\mathbf{x}}_{2}, t\right)=\epsilon^{2 k} P\left(\mathbf{x}_{1}, \mathbf{x}_{2}, t\right) .
$$

The original domain $\Omega$ is mapped into the rescaled domain $\omega$.

(4) Apply the effective domain transformation: The $\mathcal{T}_{2}$ transformation to reduce the original $d$-dimensional problem to a $d_{e}$-dimensional problem (cf. Subsec. A.2 requires the following rescaled densities

$$
\hat{p}_{e}\left(\hat{x}_{1}, t\right)=\Lambda \hat{p}\left(\hat{\mathbf{x}}_{1}, t\right), \quad \hat{P}_{e}\left(\hat{x}_{1}, \hat{x}_{2}, t\right)=\Lambda^{2} \hat{P}\left(\hat{\mathbf{x}}_{1}, \hat{\mathbf{x}}_{2}, t\right),
$$

where

$$
\Lambda=\prod_{i=1}^{k} h_{i}
$$

This factor is, in fact, equal to the volume of the rescaled domain $\omega$ (also $|\omega| \equiv\left|\omega_{c}\right|$ ). Recall it is introduced so that $\hat{p}_{e}$ and $\hat{P}_{e}$ are defined as densities. 
(5) Evaluate the collision integral $\mathcal{I}$ : The evaluation of the contribution of the two-particle interaction $\mathcal{I}$ reduces to computing one coefficient like $M_{1}(\boldsymbol{h})$ in [57] for each of the unconfined dimensions. Suppose that all the unconfined dimensions are symmetric. In general it is equal to

$$
M_{1}(\boldsymbol{h})=\int_{\omega_{c}} \mu_{1}\left(\boldsymbol{h}, \hat{\boldsymbol{x}}_{c}\right) \mathrm{d} \hat{\boldsymbol{x}}_{c},
$$

where $\hat{\boldsymbol{x}}_{c}$ are the confined coordinates of the first particle (it was simply $\hat{y}$ in the (NC2) [cf. (55)]. The function $\mu_{1}\left(\boldsymbol{h}, \hat{\boldsymbol{x}}_{c}\right)$ is the integral of $\hat{x}^{2}$ over the contact surface between two particles when the first one has coordinates $\left(\hat{\boldsymbol{x}}_{e}, \hat{\boldsymbol{x}}_{c}\right)$. Without loss of generality we can set $\hat{\boldsymbol{x}}_{e} \equiv \mathbf{0}_{e}$. In the rescaled problem, this surface is a $d$-dimensional unit sphere centred at $\left(\mathbf{0}_{e}, \hat{\boldsymbol{x}}_{c}\right)$, and (possibly) intersected with the confinement walls $\partial \omega_{c}$ :

$$
\mu_{1}\left(\boldsymbol{h}, \hat{\boldsymbol{x}}_{c}\right)=\int_{B\left(\hat{\boldsymbol{x}}_{c}\right) \cap \omega_{c}} x^{2} \mathrm{~d} S,
$$

where $B\left(\hat{\boldsymbol{x}}_{c}\right)$ is the unit ball, $\mathrm{d} S$ is the surface differential and $x$ is the unconfined dimension of this surface. Once $M_{1}$ is computed, we use it for the nonlinear coefficient of the effective Fokker-Planck equation. The generalisation of the coefficient $\alpha_{h}$ in (10) is given by

$$
\alpha_{\boldsymbol{h}}=\frac{1}{\Lambda} M_{1}(\boldsymbol{h}) .
$$

\section{References}

Ackerson BJ, Fleishman L (1982) Correlations for dilute hard core suspensions. J Chem Phys $76: 2675-2679$.

Alberts B, Johnson A, Lewis J, Raff M, Roberts K, Walter P (2002) Molecular biology of the cell. New York: Garland Science.

Bodnar M, Velázquez JJL (2005) Derivation of macroscopic equations for individual cell-based models: a formal approach. Math Meth Appl Sci 28(15):1757-1779.

Bruna M (2012) Excluded-volume Effects in Stochastic Models of Diffusion. DPhil thesis, University of Oxford.

Bruna M, Chapman SJ (2012a) Diffusion of multiple species with excluded-volume effects. J Chem Phys 137(20):204,116-204,116-16.

Bruna M, Chapman SJ (2012b) Excluded-volume effects in the diffusion of hard spheres. Phys Rev E 85(1):011103.

Burada PS, Hänggi P, Schmid G, Talkner P (2009) Diffusion in Confined Geometries. ChemPhysChem 10(1):45-54.

Carrillo JA, McCann RJ, Villani C (2003) Kinetic equilibration rates for granular media and related equations: entropy dissipation and mass transportation estimates. Rev Mat Iberoam 19(3):971-1018.

Chib S, Greenberg E (1995) Understanding the metropolis-hastings algorithm. Am Stat 49:327-335.

Dekker C (2007) Solid-state nanopores. Nat Nanotechnol 2(4):209-215

Eichhorn R, Reimann P, Hänggi P (2002) Brownian Motion Exhibiting Absolute Negative Mobility. Phys Rev Lett 88(19):190601.

Erban R, Chapman SJ, Maini PK (2007) A practical guide to stochastic simulations of reactiondiffusion processes. Arxiv preprint arXiv:07041908.

Hänggi P, Marchesoni F (2009) Artificial Brownian motors: Controlling transport on the nanoscale. Rev Mod Phys 81(1):387.

Henle ML, DiDonna B, Santangelo CD, Gopinathan A (2008) Diffusion and binding of finitesize particles in confined geometries. Phys Rev E 78(3):031118.

Hille B (2001) Ion Channels of Excitable Membranes. Sinauer, Sunderland, MA.

Howorka S, Siwy Z (2009) Nanopore analytics: sensing of single molecules. Chem Soc Rev $38(8): 2360-2384$.

Jacobs MH (1967) Diffusion Processes. Springer-Verlag, New York.

Keil FJ, Krishna R, Coppens MO (2000) Modeling of Diffusion in Zeolites. Rev Chem Eng 16(2):71-197. 
Klumpp S, Nieuwenhuizen TM, Lipowsky R (2005) Movements of molecular motors: Ratchets, random walks and traffic phenomena. Physica E 29(1-2):380-389.

Kolomeisky AB, Fisher ME (2007) Molecular Motors: A Theorist's Perspective. Annu Rev Ecol Evol Syst 58(1):675-695.

Lizana L, Ambjörnsson T (2009) Diffusion of finite-sized hard-core interacting particles in a one-dimensional box: Tagged particle dynamics. Phys Rev E 80(5):051103.

Muñoz-Gutiérrez E, Alvarez-Ramírez J, Dagdug L, Espinosa-Paredes G (2012) Diffusion in one-dimensional channels with zero-mean time-periodic tilting forces. J Chem Phys 136(11):114103.

Nicolau Jr DV, Hancock JF, Burrage K (2007) Sources of anomalous diffusion on cell membranes: a Monte Carlo study. Biophys J 92(6):1975-1987.

Plank MJ, Simpson MJ (2012) Models of collective cell behaviour with crowding effects: comparing lattice-based and lattice-free approaches. J R Soc Interface 9(76):2983-2996.

Pries AR, Secomb TW, Gaehtgens P (1996) Biophysical aspects of blood flow in the microvasculature. Cardiovasc Res 32(4):654-667.

Reguera D, Rubí J (2001) Kinetic equations for diffusion in the presence of entropic barriers. Phys Rev E 64(6):061106.

Reimann P (2002) Brownian motors: noisy transport far from equilibrium. Phys Rep 361(2):57265.

Riefler W, Schmid G, Burada PS, Hänggi P (2010) Entropic transport of finite size particles. J Phys: Condens Matter 22(45):454109.

Rost H (1984) Diffusion de sphéres dures dans la droite réelle: Comportement macroscopique et equilibre local. In: Azéma J, Yor M (eds) Séminaire de Probabilités XVIII 1982/83, Springer Berlin, pp 127-143.

Rubinstein J, Keller JB (1989) Particle distribution functions in suspensions. Phys Fluids A A1(10):1632-1641.

Scala A, Voigtmann T, De Michele C (2007) Event-driven Brownian dynamics for hard spheres. J Chem Phys 126(13):134109.

Slater GW, Guo HL, Nixon GI (1997) Bidirectional transport of polyelectrolytes using selfmodulating entropic ratchets. Phys Rev Lett 78(6):1170-1173.

Trefethen LN, others (2011) Chebfun Version 4.2. The Chebfun Development Team.

Zwanzig R (1992) Diffusion past an entropy barrier. J Phys Chem 96(10):3926-3930. 